\title{
Annihilation-in-flight of polarised positrons with polarised electrons as an analyser of the positron polarisation from muon decay
}

\author{
W. Fetscher ${ }^{\mathrm{a}}$ \\ Institute for Particle Physics (IPP), ETH Zürich, HPK G 30, Schafmattstr. 20, 8093 Zürich, Switzerland
}

Received: 26 April 2007 /

Published online: 3 August 2007 - (C) Springer-Verlag / Società Italiana di Fisica 2007

\begin{abstract}
The use of annihilation-in-flight of arbitrarily polarised positrons with arbitrarily polarised electrons as an analyser for the positron polarisation from muon decay is discussed. Analysing powers for the longitudinal and the two transverse positron polarisation components are derived and algorithms for the simulation of polarised muon decay and of annihilation-in-flight are given.
\end{abstract}

PACS. 13.88.+e; 13.35.Bv; 12.60.-i; 11.30.Er; 02.70.Uu

\section{Introduction}

The chiral nature of the charged weak interaction manifests itself through the appearance of pseudoscalar terms like decay asymmetries and lepton longitudinal polarisations both of which involve measurements of particle spins. The corresponding measurements in nuclear $\beta$ decay [1], pion and muon decay $[2-4]$ proved that the charged weak interactions prefers leptons of negative helicity and antileptons of positive helicity. This experimental evidence has led to the concept of lefthanded fermions ( $V-A$ hypothesis) and the standard model [5-7], which has been extremely successful in describing experimental data. Still, the question remains, if extensions to the standard model are needed. Effects of additional, weaker, interactions could be found with the help of precision measurements.

These investigations can be pursued especially well in the case of muon decay. It can be described by the most general, local four-fermion point interaction Hamiltonian [8]. It contains ten complex coupling constants to be determined by experiment. The observables can be expressed in terms of a chiral Hamiltonian in chargechanging form characterized by fields of definite handedness [9]. The matrix element is given by $[10,11]$ :

$$
\mathcal{M}=\frac{4 G_{\mathrm{F}}}{\sqrt{2}} \sum_{\gamma, \varepsilon, \mu} g_{\varepsilon \mu}^{\gamma}\left\langle\bar{e}_{\varepsilon}\left|\Gamma^{\gamma}\right|\left(\nu_{e}\right)_{n}\right\rangle\left\langle\bar{\nu}_{m}\left|\Gamma_{\gamma}\right|(\mu)_{\mu}\right\rangle
$$

The index $\gamma=\mathrm{S}, \mathrm{V}, \mathrm{T}$ labels the type of interaction (4scalar, 4-vector, 4 -tensor), while the indices $\varepsilon, \mu=\mathrm{R}, \mathrm{L}$ indicate the chirality (R, L: right-, lefthanded) of the particle spinors. The standard model predicts $g_{\mathrm{LL}}^{\mathrm{V}}=1$, with all

\footnotetext{
a e-mail: fetscher@phys.ethz.ch
}

other couplings being zero. In fact, with a selected set of $\mu$ decay experiments it has been possible to derive a lower limit for $g_{\mathrm{LL}}^{\mathrm{V}}$ and upper limits for the absolute values of all other 9 coupling constants [10].

The chiral nature of the weak interaction implies the special importance of spin-dependent measurements as decay asymmetries and polarisation measurements, while measurements of scalar quantities as the Michel parameter $\rho$ which parametrizes the energy spectrum of the decay positrons do not constrain the possible interactions sufficiently. In fact, a precise measurement of $\rho$ in agreement with the standard model prediction $\rho=3 / 4$ does not imply the $V-A$ interaction. Indeed, it has been shown that any linear combination of the six complex coupling constants $g_{\mathrm{RR}}^{\mathrm{S}}, g_{\mathrm{RL}}^{\mathrm{S}}, g_{\mathrm{LR}}^{\mathrm{S}}, g_{\mathrm{LL}}^{\mathrm{S}}, g_{\mathrm{RR}}^{\mathrm{V}}$, and $g_{\mathrm{LL}}^{\mathrm{V}}$ results in $\rho=3 / 4[12]$.

A measurement of the longitudinal polarisation (decay parameter $\xi^{\prime}$ ), on the other hand, allows to derive stringent limits to five of the ten complex coupling constants, if it turns out to be in agreement with the standard model value of 1 , within errors $[12,13]$ :

$$
\begin{aligned}
& \frac{1}{4}\left|g_{\mathrm{RR}}^{\mathrm{S}}\right|^{2}+\frac{1}{4}\left|g_{\mathrm{RL}}^{\mathrm{S}}\right|^{2}+\left|g_{\mathrm{RR}}^{\mathrm{V}}\right|^{2}+\left|g_{\mathrm{RL}}^{\mathrm{V}}\right|^{2}+3\left|g_{\mathrm{RL}}^{\mathrm{T}}\right|^{2} \\
& =\frac{1}{2}\left(1-\xi^{\prime}\right) .
\end{aligned}
$$

Note that because the left-hand side of (2) contains the sum of positive squares, the standard model value of exactly $\xi^{\prime}=1$ excludes any righthanded contributions for the electron (first lower index of the coupling constant).

In striking contrast to that, the measurement of possible transverse polarisation components does not allow to derive such stringent limits, because any sizeable transverse component is due to interference between different 
interactions, so that the resulting amplitude may well be negative. The energy dependence of the transverse component $P_{\mathrm{T}_{1}}$, which lies in the plane of the muon polarisation and positron momentum, is described by the decay parameters $\eta$ and $\eta^{\prime \prime}[11,14]$ or, in the older publications, by $\alpha / A$ and $\beta / A[15,16]$. The energy dependence of the transverse component $P_{\mathrm{T}_{2}}$, which lies perpendicular to the mentioned plane, is described by the decay parameters $\alpha^{\prime} / A$ and $\beta^{\prime} / A$. In terms of the coupling constants of (1) these four parameters are given by:

$$
\begin{aligned}
\eta= & \frac{1}{2} \operatorname{Re}\left\{g_{\mathrm{LL}}^{\mathrm{V}} g_{\mathrm{RR}}^{\mathrm{S} *}+g_{\mathrm{RR}}^{\mathrm{V}} g_{\mathrm{LL}}^{\mathrm{S} *}+g_{\mathrm{LR}}^{\mathrm{V}}\left(g_{\mathrm{RL}}^{\mathrm{S} *}+6 g_{\mathrm{RL}}^{\mathrm{T} *}\right)\right. \\
& \left.+g_{\mathrm{RL}}^{\mathrm{V}}\left(g_{\mathrm{LR}}^{\mathrm{S} *}+6 g_{\mathrm{LR}}^{\mathrm{T} *}\right)\right\} \\
\eta^{\prime \prime}= & \frac{1}{2} \operatorname{Re}\left\{-g_{\mathrm{LL}}^{\mathrm{V}} g_{\mathrm{RR}}^{\mathrm{S} *}-g_{\mathrm{RR}}^{\mathrm{V}} g_{\mathrm{LL}}^{\mathrm{S} *}+3 g_{\mathrm{LR}}^{\mathrm{V}}\left(g_{\mathrm{RL}}^{\mathrm{S} *}+6 g_{\mathrm{RL}}^{\mathrm{T} *}\right)\right. \\
& \left.+3 g_{\mathrm{RL}}^{\mathrm{V}}\left(g_{\mathrm{LR}}^{\mathrm{S} *}+6 g_{\mathrm{LR}}^{\mathrm{T} *}\right)\right\} \\
\frac{\alpha^{\prime}}{A}= & \frac{1}{2} \operatorname{Im}\left\{g_{\mathrm{LR}}^{\mathrm{V}}\left(g_{\mathrm{RL}}^{\mathrm{S} *}+6 g_{\mathrm{RL}}^{\mathrm{T} *}\right)+g_{\mathrm{RL}}^{\mathrm{V}}\left(g_{\mathrm{LR}}^{\mathrm{S} *}+6 g_{\mathrm{LR}}^{\mathrm{T} *}\right)\right\} \\
\frac{\beta^{\prime}}{A}= & \frac{1}{4} \operatorname{Im}\left\{g_{\mathrm{RR}}^{\mathrm{V}} g_{\mathrm{LL}}^{\mathrm{S} *}-g_{\mathrm{LL}}^{\mathrm{V}} g_{\mathrm{RR}}^{\mathrm{S} *}\right\} .
\end{aligned}
$$

In contrast to our conclusions for the precise measurement of $P_{\mathrm{L}}$, i.e., the $\xi^{\prime}$ parameter, a precise measurement of the four parameters $\eta, \eta^{\prime \prime}, \alpha^{\prime} / A$ and $\beta^{\prime} / A$ does not permit to exclude any coupling constants, since in the standard model all four parameters are equal to zero, and as interference terms, they can cancel each other mutually. However, with our knowledge that $g_{\mathrm{LL}}^{\mathrm{V}}>0.96(90 \%$ c.l. $)[10,11]$, we can neglect terms in second order of the nine complex couplings consistent with zero. With the approximation $g_{\mathrm{LL}}^{\mathrm{V}} \approx 1$ we obtain:

$$
\begin{aligned}
\eta & =\frac{1}{2} \operatorname{Re}\left\{g_{\mathrm{RR}}^{\mathrm{S}}\right\} \\
\eta^{\prime \prime} & =-\frac{1}{2} \operatorname{Re}\left\{g_{\mathrm{RR}}^{\mathrm{S}}\right\} \\
\frac{\alpha^{\prime}}{A} & =0 \\
\frac{\beta^{\prime}}{A} & =\frac{1}{4} \operatorname{Im}\left\{g_{\mathrm{RR}}^{\mathrm{S}}\right\} .
\end{aligned}
$$

The specific advantage of the measurement of the transverse components here becomes obvious: One is able to measure the scalar coupling $g_{\mathrm{RR}}^{\mathrm{S}}$ linearly and, since $P_{\mathrm{T}_{2}}$ violates time reversal invariance, one gets separate limits for the real and for the imaginary part of this coupling constant with a corresponding gain in sensitivity as compared to the measurements of the longitudinal polarisation $P_{\mathrm{L}} \pm$ $\Delta P_{\mathrm{L}}$. This would yield $\Delta \xi^{\prime} / \xi^{\prime}=\Delta P_{\mathrm{L}} / P_{\mathrm{L}}$ and give the following limit to $g_{\mathrm{RR}}^{\mathrm{S}}$ according to $(2)$ :

$$
\left|g_{\mathrm{RR}}^{\mathrm{S}}\right| \leq \sqrt{2 \Delta \xi^{\prime}}
$$

since $P_{\mathrm{L}} \approx 1$. This obviously is not as sensitive as the direct measurement of the transverse polarisation due to the proportionality to $\sqrt{\Delta P_{\mathrm{L}}}$. The argument above is equivalent to the relation

$$
\sqrt{P_{\mathrm{T}_{1}}^{2}+P_{\mathrm{T}_{2}}^{2}} \leq \sqrt{1-P_{\mathrm{L}}^{2}} \approx \sqrt{2 \Delta P_{\mathrm{L}}}
$$

which follows from the definition of the polarisation, $|\mathbf{P}| \leq 1$.

Moreover, a measurement of $P_{\mathrm{L}}$, as an absolute measurement, is quite difficult due to the small available electron polarisation of $7 \%$. The measurement of $P_{\mathrm{T}_{1}}$ and $P_{\mathrm{T}_{2}}$ is simplified by the fact that both components are small or zero, so that the experimental errors are of the same order of magnitude as the values themselves and therefore much less critical than in the case of $P_{\mathrm{L}}$.

For intermediate energies (a few $\mathrm{MeV}$ up to $\approx 53 \mathrm{MeV}$ ) the annihilation-in-flight of positrons with polarised electrons in a magnetised foil has been shown to efficiently analyse both the transverse $[17,18]$ and the longitudinal [19-21] polarisation. The cross section in the c.m. system for this process had been calculated already in the late 1950's and early 1960's [22,23]. From this cross section the analysing power for the longitudinal polarization has been derived $[19,24]$.

The experimental method for the measurement of the transverse polarisation is identical for both measurements performed up to date. The first experiment at PSI (PSIPT I) used four big NaI detectors as a calorimeter [17]. With a slightly modified setup the longitudinal polarisation was also measured [20,21]. The second experiment (PSIPT II) used parts of the same setup, but, among other improvements, replaced the four NaI detectors by 127 BGO crystals $[18,25]$. Since the measurement of $P_{\mathrm{T}}$ makes use of a precessing muon polarisation vector (see Sect. 5.1), the determination of the respective analysing powers turns out to be quite demanding. They depend not only on the energies of the positron and of the two annihilation quanta, but also on the azimuthal angle of orientation $\psi$ of the gamma rays (for the definition of $\psi$ see Figs. 2 and 4). For PSIPT I these analysing powers were derived for positrons emitted in the direction of the symmetry axis of the setup and for the angles $\psi=0^{\circ}, 45^{\circ}, 90^{\circ}$, and $135^{\circ}$, corresponding to the four possible orientations of NaI detector pairs [26].

For PSIPT II, with its finegrained BGO detector wall, $\psi$ was measured for each event separately. In addition to that, the data analysis was performed by comparing the measured data with Monte Carlo generated polarisation distributions, one for each of the four decay parameters $\eta$, $\eta^{\prime \prime}, \alpha^{\prime} / A$ and $\beta^{\prime} / A[27]$. For this simulation the emission of positrons of arbitrary polarisation and emitted into arbitrary directions from the rotating coordinate system of the muon has been calculated. The dependence of the annihilation rate on time as well as on the azimuthal angle $\alpha$ and the polar angle $\chi$ with respect to the fixed axes of the experiment is derived for the first time, thereby including all three polarisation components (106). As a by-product of this result, a new possible method to measure the longitudinal polarisation is presented.

This comprehensive overview of the theory underlying this kind of experiment generalises previous results, as used for PSIPT I, by deriving distributions and analysing powers for arbitrary polarisation values and directions of emission of the positrons. It establishes the relations between coupling constants, decay parameters and polarisation components. It also clarifies the hitherto neglected 
difference between $P_{\mathrm{T}_{1}}(x, \vartheta)$, as predicted by theory, and $P_{1}(x)$, as obtained in the experiment. Finally it derives algorithms to simulate polarised muon decay and the polarisation dependent annihilation.

\section{Polarisation vector of the decay positron}

The polarisation vector of the $e^{+}$is described most easily in the coordinate system defined by the muon polarisation vector $\mathbf{P}_{\mu}$ and the positron momentum $\mathbf{k}_{e}$ (see Fig. 1):

$$
\mathbf{P}_{e^{+}}=P_{\mathrm{T}_{1}}(\vartheta, x) \hat{\boldsymbol{x}}_{0}+P_{\mathrm{T}_{2}}(\vartheta, x) \hat{\boldsymbol{y}}_{0}+P_{\mathrm{L}} \hat{\boldsymbol{z}}_{0},
$$

Here, $\vartheta$ is the angle between positron momentum $\mathbf{k}_{e}$ and muon polarisation $\mathbf{P}_{\mu}$, and $x$ is the reduced positron energy:

$$
x_{0} \equiv \frac{m_{e}}{W_{\mu e}} \leq x=\frac{E_{e}}{W_{\mu e}} \leq 1,
$$

with $W_{\mu e}=\left(m_{\mu}^{2}+m_{e}^{2}\right) /\left(2 m_{\mu}\right)$ as the maximum energy of the positron. The orthogonal unit vectors $\hat{\boldsymbol{x}}_{0}, \hat{\boldsymbol{y}}_{0}, \hat{\boldsymbol{z}}_{0}$ are defined as follows:

$$
\begin{aligned}
& \hat{\boldsymbol{z}}_{0}=\frac{\mathbf{k}_{e}}{\left|\mathbf{k}_{e}\right|} \\
& \hat{\boldsymbol{y}}_{0}=\frac{\hat{\boldsymbol{z}}_{0} \times \mathbf{P}_{\mu}}{\left|\hat{\boldsymbol{z}}_{0} \times \mathbf{P}_{\mu}\right|} \\
& \hat{\boldsymbol{x}}_{0}=\hat{\boldsymbol{y}}_{0} \times \hat{\boldsymbol{z}}_{0} .
\end{aligned}
$$

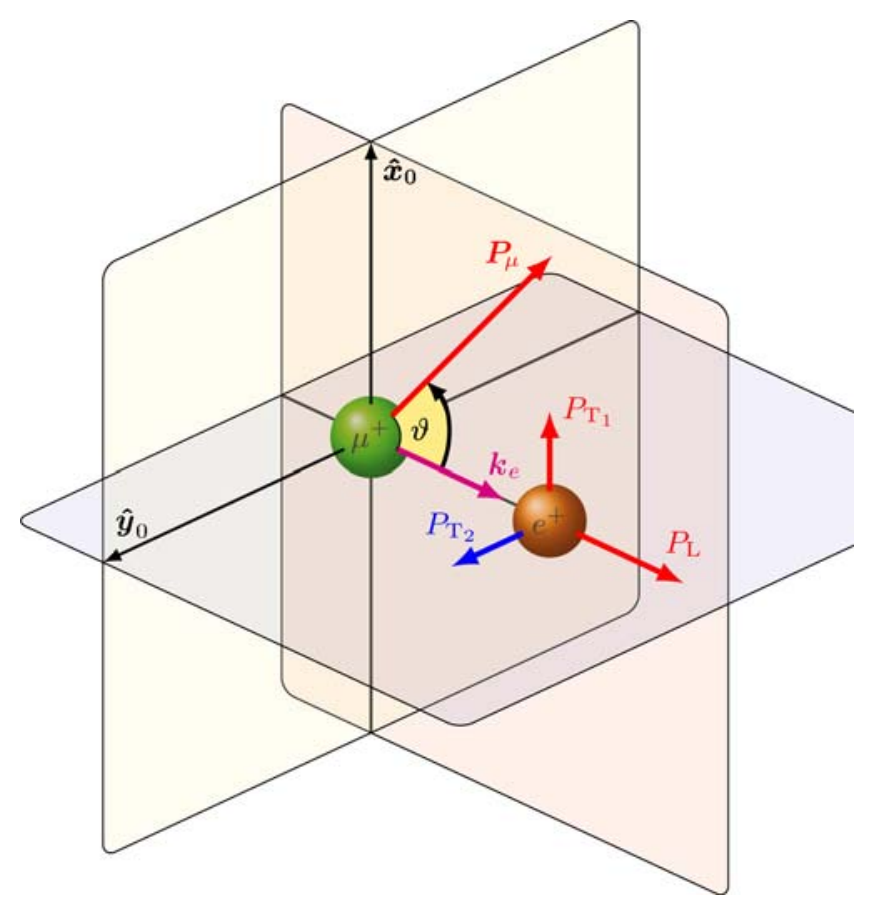

Fig. 1. Definition of the observables in polarised muon decay: muon polarisation $\mathbf{P}_{\mu}$, positron momentum $\mathbf{k}_{e}$, longitudinal positron polarisation $P_{\mathrm{L}}$, transverse positron polarisation $\left(P_{\mathrm{T}_{1}}, P_{\mathrm{T}_{2}}\right)$ and angle of emission $\vartheta$ (relative to $\left.\mathbf{P}_{\mu}\right)$. Time reversal invariance is violated if $P_{\mathrm{T}_{2}} \neq 0$
The components of $\mathbf{P}_{e}$ then are given by [11,14]:

$$
\begin{aligned}
P_{\mathrm{T}_{1}}(x, \vartheta) & =\frac{P_{\mu} \sin \vartheta F_{\mathrm{T}_{1}}(x)}{F_{\mathrm{IS}}(x)+P_{\mu} \cos \vartheta F_{\mathrm{AS}}(x)} \\
P_{\mathrm{T}_{2}}(x, \vartheta) & =\frac{P_{\mu} \sin \vartheta F_{\mathrm{T}_{2}}(x)}{F_{\mathrm{IS}}(x)+P_{\mu} \cos \vartheta F_{\mathrm{AS}}(x)} \\
P_{\mathrm{L}}(x, \vartheta) & =\frac{F_{\mathrm{IP}}(x)+P_{\mu} \cos \vartheta F_{\mathrm{AP}}(x)}{F_{\mathrm{IS}}(x)+P_{\mu} \cos \vartheta F_{\mathrm{AS}}(x)} .
\end{aligned}
$$

The functions $F_{i}(x)$ are given by:

$$
\begin{aligned}
F_{\mathrm{IS}}(x)= & x(1-x)+\frac{2}{9} \rho\left(4 x^{2}-3 x-x_{0}^{2}\right)+\eta x_{0}(1-x) \\
F_{\mathrm{AS}}(x)= & \frac{1}{3} \xi \sqrt{x^{2}-x_{0}^{2}} \\
& \times\left\{1-x+\frac{2}{3} \delta\left[4 x-3-\left(\sqrt{1-x_{0}^{2}}-1\right)\right]\right\} \\
F_{\mathrm{T}_{1}}(x)= & \frac{1}{12}\left\{-2\left[\xi^{\prime \prime}+12\left(\rho-\frac{3}{4}\right)\right](1-x) x_{0}\right. \\
& \left.-3 \eta\left(x^{2}-x_{0}^{2}\right)+\eta^{\prime \prime}\left(-3 x^{2}+4 x-x_{0}^{2}\right)\right\} \\
F_{\mathrm{T}_{2}}(x)= & \frac{1}{3} \sqrt{x^{2}-x_{0}^{2}}\left\{3 \frac{\alpha^{\prime}}{A}(1-x)+2 \frac{\beta^{\prime}}{A} \sqrt{1-x_{0}^{2}}\right\} \\
F_{\mathrm{IP}}(x)= & \frac{1}{54} \sqrt{x^{2}-x_{0}^{2}}\left\{9 \xi^{\prime}\left(-2 x+2+\sqrt{1-x_{0}^{2}}\right)\right. \\
& \left.+4 \xi\left(\delta-\frac{3}{4}\right)\left(4 x-4+\sqrt{1-x_{0}^{2}}\right)\right\} \\
F_{\mathrm{AP}}(x)= & \frac{1}{6}\left\{\xi^{\prime \prime}\left(2 x^{2}-x-x_{0}^{2}\right)+2 \eta^{\prime \prime}(1-x) x_{0}\right. \\
& \left.+4\left(\rho-\frac{3}{4}\right)\left(4 x^{2}-3 x-x_{0}^{2}\right)\right\}
\end{aligned}
$$

with $\xi^{\prime \prime}=1+\operatorname{Re}\left\{g_{\mathrm{RL}}^{\mathrm{V}}\left(g_{\mathrm{LR}}^{\mathrm{S} *}+6 g_{\mathrm{LR}}^{\mathrm{T} *}\right)+g_{\mathrm{LR}}^{\mathrm{V}}\left(g_{\mathrm{RL}}^{\mathrm{S} *}+6 g_{\mathrm{RL}}^{\mathrm{T} *}\right)\right\}$

$$
-\frac{5}{8}\left\{\left|g_{\mathrm{RL}}^{\mathrm{S}}-2 g_{\mathrm{RL}}^{\mathrm{T}}\right|^{2}+\left|g_{\mathrm{LR}}^{\mathrm{S}}-2 g_{\mathrm{LR}}^{\mathrm{T}}\right|^{2}\right\} \text {. }
$$

In the standard model, $\xi^{\prime}=\xi^{\prime \prime}=1$. Radiative corrections to the polarisation observables have been calculated recently including terms with $x_{0}$ [28]. Their influence, however, is only substantial towards the soft end of the $e^{+}$spectrum. Neglecting terms proportional to $x_{0}=9.67 \times 10^{-3}$ and using the canonical $(V-A)$ values for the experimentally well-determined decay parameters $\rho=3 / 4[29], \delta=$ $3 / 4[30]$ and $\xi=1[31-33]$, I obtain

$$
\begin{aligned}
F_{\mathrm{IS}}(x) & =\frac{1}{6}\left\{-2 x^{2}+3 x\right\} \\
F_{\mathrm{AS}}(x) & =\frac{1}{6}\left\{2 x^{2}-x\right\} \\
F_{\mathrm{T}_{1}}(x) & =\frac{1}{12}\left\{-3 \eta x^{2}+\eta^{\prime \prime}\left(-3 x^{2}+4 x\right)\right\} \\
F_{\mathrm{T}_{2}}(x) & =\frac{1}{3}\left\{3 \frac{\alpha^{\prime}}{A}\left(x-x^{2}\right)+2 \frac{\beta^{\prime}}{A} x\right\}
\end{aligned}
$$




$$
\begin{gathered}
F_{\mathrm{IP}}(x)=\frac{1}{6} \xi^{\prime}\left(-2 x^{2}+3 x\right) \\
F_{\mathrm{AP}}(x)=\frac{1}{6} \xi^{\prime \prime}\left(2 x^{2}-x\right)
\end{gathered}
$$

According to (18) and (19) the transverse polarisation depends on the polar emission angle $\vartheta$ both in the numerator and in the denominator. In the experimental setup described below $\vartheta$ is distributed symmetrically around $\vartheta=$ $90^{\circ}$. There the polarisation is determined by summing over events with different $\vartheta$ which eliminates the (already small) $\vartheta$ dependence in the denominator. As a result of this procedure both components can be factorized as follows:

$$
\begin{aligned}
& P_{\mathrm{T}_{1}}(x, \vartheta)=P_{\mu} \sin \vartheta \frac{F_{\mathrm{T}_{1}}(x)}{F_{\mathrm{IS}}(x)} \equiv P_{\mu} \sin \vartheta P_{1}(x) \\
& P_{\mathrm{T}_{2}}(x, \vartheta)=P_{\mu} \sin \vartheta \frac{F_{\mathrm{T}_{2}}(x)}{F_{\mathrm{IS}}(x)} \equiv P_{\mu} \sin \vartheta P_{2}(x)
\end{aligned}
$$

The energy distributions $P_{1}(x)$ and $P_{2}(x)$ contain the full dependence on the decay parameters $\left(\eta, \eta^{\prime \prime}\right)$ and $\left(\alpha^{\prime} / A, \beta^{\prime} / A\right)$, respectively, and thus form the basis for the determination of these four decay parameters.

\section{Kinematics of annihilation-in-flight}

We regard the reaction

$$
e^{+} e^{-} \rightarrow \gamma \gamma
$$

It is convenient to use reduced four-momenta $\pi_{i}$ for this reaction:

$$
\pi_{i}:=\left(\begin{array}{c}
\varepsilon_{i} \\
\boldsymbol{\kappa}_{i}
\end{array}\right) \equiv \frac{1}{m}\left(\begin{array}{c}
E_{i} \\
\mathbf{k}_{i}
\end{array}\right) \quad(i=1, \ldots, 4),
$$

where the particles' energy and three-momentum are given by $E_{i}$ and $\mathbf{k}_{i}$, respectively, and $m$ designates the positron mass. Table 1 lists the reduced four-momenta of the particles of the annihilation reaction (36) in the laboratory and in the c.m. system.

The center of momentum (c.m.) of the positronelectron system moves with the reduced velocity

$$
\beta=\sqrt{\frac{\varepsilon_{1}-1}{\varepsilon_{1}+1}},
$$

with the associated $\gamma$-factor

$$
\gamma=\sqrt{\frac{\varepsilon_{1}+1}{2}} .
$$

The absolute value of the reduced momentum of each photon in the c.m. system is given by

$$
\kappa^{*}=\gamma
$$

We designate the photon with the higher energy in the Lab system as particle \# 3. It is emitted in the c.m. system under the polar angle $\vartheta^{*}$ and the azimuthal angle $\varphi^{*}$. The
Table 1. Labelling of the reduced fourmomenta $\pi_{i}=p_{i} / m$ of the particles of the annihilation reaction (36) in the laboratory and in the c.m. system. The positron mass is given by $m$

\begin{tabular}{llll}
\hline Particle & $\#$ & $\pi_{\text {Lab }}$ & $\pi_{\mathrm{c} . \mathrm{m} .}$ \\
\hline$e^{+}$ & 1 & $\left(\begin{array}{c}\varepsilon_{1} \\
\boldsymbol{\kappa}_{1}\end{array}\right)$ & $\left(\begin{array}{c}\varepsilon_{1}^{*} \\
\boldsymbol{\kappa}_{1}^{*}\end{array}\right)$ \\
$e^{-}$ & 2 & $\left(\begin{array}{c}1 \\
\mathbf{0}\end{array}\right)$ & $\left(\begin{array}{c}\varepsilon_{2}^{*} \\
\boldsymbol{\kappa}_{2}^{*}\end{array}\right)$ \\
$\gamma$ & 3 & $\left(\begin{array}{c}\varepsilon_{3} \\
\boldsymbol{\kappa}_{3}\end{array}\right)$ & $\left(\begin{array}{c}\varepsilon_{3}^{*} \\
\boldsymbol{\kappa}_{3}^{*}\end{array}\right)$ \\
$\gamma$ & 4 & $\left(\begin{array}{c}\varepsilon_{4} \\
\boldsymbol{\kappa}_{4}\end{array}\right)$ & $\left(\begin{array}{c}\varepsilon_{4}^{*} \\
\boldsymbol{\kappa}_{4}^{*}\end{array}\right)$ \\
\hline
\end{tabular}

resulting momenta $\pi_{3}^{*}$ and $\pi_{4}^{*}$ of the two photons in the c.m. system are then:

$$
\begin{aligned}
& \pi_{3}^{*}=\gamma\left(\begin{array}{c}
1 \\
\sin \vartheta^{*} \cos \varphi^{*} \\
\sin \vartheta^{*} \sin \varphi^{*} \\
\cos \vartheta^{*}
\end{array}\right) \\
& \pi_{4}^{*}=\gamma\left(\begin{array}{c}
1 \\
-\sin \vartheta^{*} \cos \varphi^{*} \\
-\sin \vartheta^{*} \sin \varphi^{*} \\
-\cos \vartheta^{*}
\end{array}\right)
\end{aligned}
$$

With the definition of particle \# 3 as given above, the angular region is restricted to

$$
\begin{aligned}
& 0 \leq \vartheta^{*} \leq \frac{\pi}{2} \\
& 0 \leq \varphi^{*} \leq 2 \pi .
\end{aligned}
$$

The momenta of the two photons in the lab system as functions of the polar c.m. angle $\vartheta^{*}$ and the azimuthal c.m. (as well as lab) angle $\varphi^{*} \equiv \varphi$ are obtained by a Lorentz boost:

$$
\begin{gathered}
\pi_{3}=\gamma\left(\begin{array}{c}
\gamma\left(1+\beta \cos \vartheta^{*}\right) \\
\sin \vartheta^{*} \cos \varphi \\
\sin \vartheta^{*} \sin \varphi \\
\gamma\left(\beta+\cos \vartheta^{*}\right)
\end{array}\right) \\
\pi_{4}=\gamma\left(\begin{array}{c}
\gamma\left(1-\beta \cos \vartheta^{*}\right) \\
-\sin \vartheta^{*} \cos \varphi \\
-\sin \vartheta^{*} \sin \varphi \\
\gamma\left(\beta-\cos \vartheta^{*}\right)
\end{array}\right) .
\end{gathered}
$$

The relations between the polar lab angles $\vartheta_{3}$ and $\vartheta_{4}$ and the c.m. polar angle $\vartheta^{*}$ are:

$$
\begin{aligned}
& \cos \vartheta_{3}=\frac{\beta+\cos \vartheta^{*}}{1+\beta \cos \vartheta^{*}} \\
& \cos \vartheta_{4}=\frac{\beta-\cos \vartheta^{*}}{1-\beta \cos \vartheta^{*}}
\end{aligned}
$$


The relations between the polar lab angles $\vartheta_{3}$ and $\vartheta_{4}$ and the lab energies $\varepsilon_{3}$ and $\varepsilon_{4}$ are:

$$
\begin{aligned}
\cos \vartheta_{3} & =\frac{\varepsilon_{3}-1}{\beta \varepsilon_{3}} \\
\cos \vartheta_{4} & =\frac{\varepsilon_{4}-1}{\beta \varepsilon_{4}} .
\end{aligned}
$$

The opening angle $\vartheta_{34}$ between the lab momenta of the two annihilation quanta is:

$$
\cos \vartheta_{34}=1-\frac{\varepsilon_{3}+\varepsilon_{4}}{\varepsilon_{3} \varepsilon_{4}} .
$$

\section{Annihilation cross section for arbitrarely polarised electrons and positrons}

The matrix elements for the annihilation of arbitrarely polarised electrons and positrons have been calculated by W.H. McMaster [23]. There the polarisation vectors of the $e^{-}$and the $e^{+}$, in this paper labelled as $\mathbf{Q}$ and $\mathbf{P}$, repectively, are extended to the Stokes vectors

$$
\begin{aligned}
(1, \mathbf{Q}) & \equiv\left(1, Q_{c}, Q_{a}, Q_{b}\right) \\
\left(\begin{array}{c}
1 \\
\mathbf{P}
\end{array}\right) & \equiv\left(\begin{array}{c}
1 \\
P_{c} \\
P_{a} \\
P_{b}
\end{array}\right)
\end{aligned}
$$

where the first entry stands for the intensity of the beam, while the other three are the usual components of the polarisation, with $P_{c}, P_{a}$, and $P_{b}$ corresponding to the $z, x$, and $y$ component, respectively. The differential cross section in the c.m. system, finally, is given by

$$
\frac{\mathrm{d} \sigma}{\mathrm{d} \Omega}=(1, \mathbf{Q}) T\left(\begin{array}{l}
1 \\
\mathbf{P}
\end{array}\right),
$$

where $T$ is a $4 \times 4$ matrix as given by (69) of [23]. After correcting for an obvious error ${ }^{1}$ and summing over the unobserved polarisation states of the two annihilation quanta, we obtain:

$$
\frac{\mathrm{d} \sigma}{\mathrm{d} \Omega}=\sigma_{0}+\sigma_{x} P_{a} Q_{a}+\sigma_{y} P_{b} Q_{b}+\sigma_{z} P_{c} Q_{c} .
$$

with the abbreviations

$$
\begin{aligned}
\sigma_{0} & \equiv \frac{r_{0}^{2}}{4 \beta \gamma^{2}\left(1-\beta^{2} \cos ^{2} \vartheta^{*}\right)^{2}}[A+B+C+D] \\
\sigma_{x} & \equiv \frac{r_{0}^{2}}{4 \beta \gamma^{2}\left(1-\beta^{2} \cos ^{2} \vartheta^{*}\right)^{2}}[A+B-C-D] \\
\sigma_{y} & \equiv \frac{r_{0}^{2}}{4 \beta \gamma^{2}\left(1-\beta^{2} \cos ^{2} \vartheta^{*}\right)^{2}}[A-B+C-D] \\
\sigma_{z} & \equiv \frac{r_{0}^{2}}{4 \beta \gamma^{2}\left(1-\beta^{2} \cos ^{2} \vartheta^{*}\right)^{2}}[-A+B+C-D]
\end{aligned}
$$

1 The Term $T_{c}^{22}=H^{2}-2 H_{z}^{2}-2 H_{0}^{2}$ should be replaced by $H^{2}-2 H_{z}^{2}-H_{0}^{2}$. and

$$
\begin{aligned}
r_{0} & =\frac{1}{4 \pi \varepsilon_{0}} \frac{e^{2}}{m c^{2}}=2.818 \mathrm{fm} \\
A & \equiv \frac{\beta^{2}}{\gamma^{2}}\left(1+\sin ^{4} \vartheta^{*}\right) \\
B & \equiv \beta^{2} \sin ^{2} \vartheta^{*} \\
C & \equiv \beta^{2} \sin ^{2} \vartheta^{*} \cos ^{2} \vartheta^{*} \\
D & \equiv \frac{1}{\gamma^{2}} .
\end{aligned}
$$

\section{Annihilation as an analyser of the positron polarisation}

\subsection{Introduction}

A realistic experimental setup for the measurement of the positron polarisation consists of a polarised positron source, a magnetised foil as polarisation analyser and a highly granular electromagnetic calorimeter as detector for the annihilation quanta. This is shown in Fig. 2 which displays the experimental setup of the recently published measurement of the transverse positron polarisation from muon decay $[18,25]$.

The measurement of the two transverse positron polarisation components $P_{\mathrm{T}_{1}}$ and $P_{\mathrm{T}_{2}}$ relies on the fact that the annihilation cross section for transversely polarised $e^{+}$ with transversely polarised $e^{-}$leads to an anisotropic azimuthal distribution of the two annihilation quanta, as will be shown below. By precessing the muon polarisation in a homogeneous magnetic field the transverse polarisation will precess with the same angular frequency $\omega$. This, in turn, leads to a corresponding rotation of the annihilation quanta distribution. It finally results in a harmonic variation of the annihilation rate of a given detector pair with azimuthal orientation $\psi$. Neglecting effects of multiple scattering I will deduce the amplitude and phase of this annihilation signal. The effects of multiple scattering will only effect the amplitude, but not the general form thus obtained. This change can be readily obtained by a Monte Carlo simulation specific to a given experiment.

The following calculation, although performed specifically for the derivation of the two transverse components, includes also the longitudinal polarisation. In fact, it will be shown that it can be applied just as well to a measurement of $P_{\mathrm{L}}$, for which a rotation of $\mathbf{P}_{\mu}$ is not required. Indeed, measurements of $P_{\mathrm{L}}$ prefer to use an unpolarised sample of muons $[20,21,24]$, unless one wishes to measure the angular dependence of $P_{\mathrm{L}}$ as the experiment of the Louvain-la-Neuve-PSI-ETH collaboration does [34].

\subsection{Global and local coordinate systems}

For the description of the experiment I use a series of righthanded coordinate systems, each of them adapted to a special purpose: 


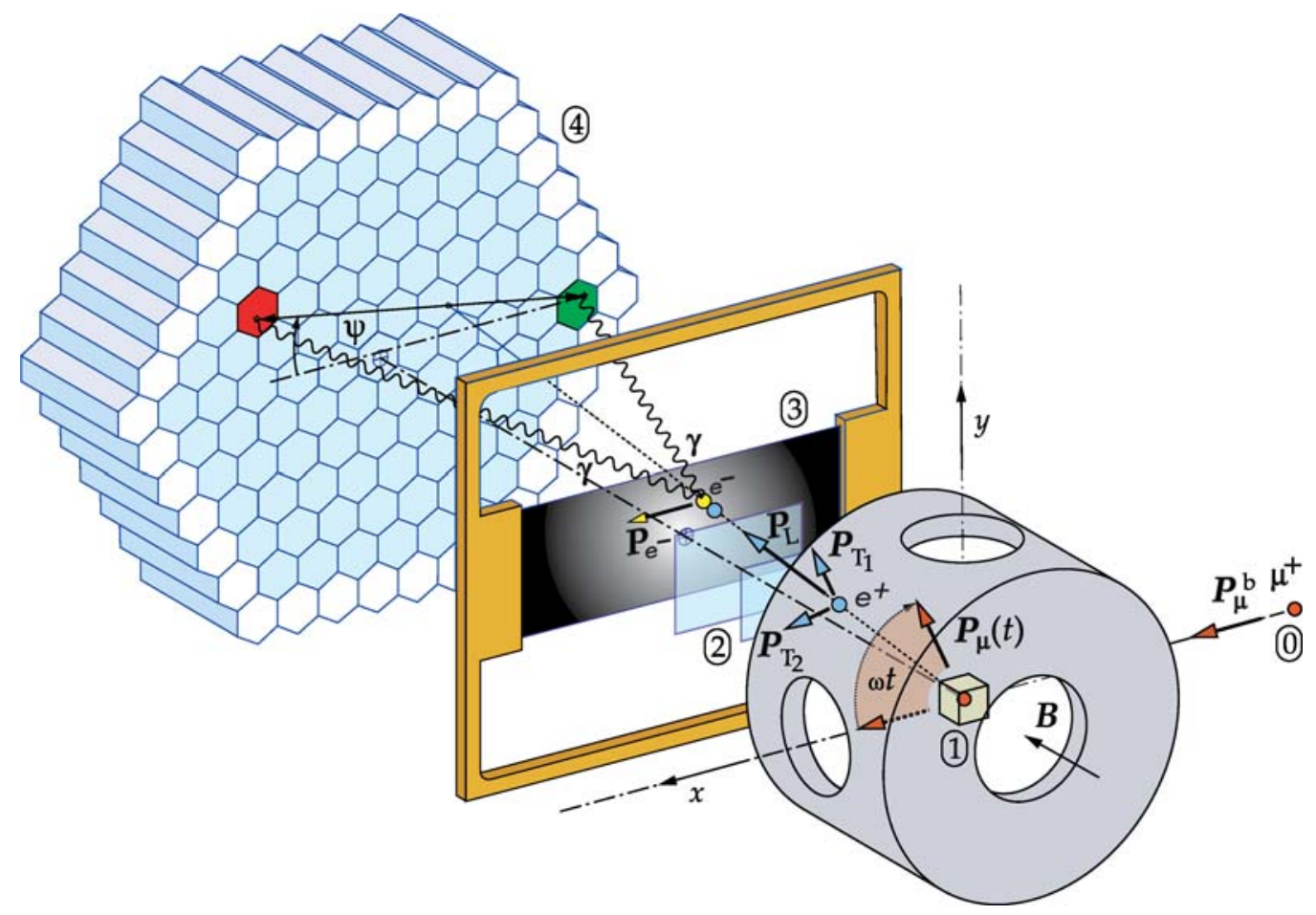

Fig. 2. Schematic view of the experimental setup of Danneberg et al. [18]. 0: burst of polarised muons (angular frequency $\omega$, polarisation $\left.P_{\mu}^{\mathrm{b}}\right)$; 1 : be stop target and precession field $B ; 2$ : two plastic scintillation counters selecting decay positrons; 3 : magnetised Vacoflux 50 foil serving as polarisation analyser; 4: array of 127 BGO scintillators to detect the two $\gamma^{\prime}$ s from $e^{+}$annihilation-inflight

1. The global coordinate system $\mathbf{K}$ with base vectors $\hat{\boldsymbol{x}}, \hat{\boldsymbol{y}}$, and $\hat{z}$ as shown in Fig. 2. Vectors given in component form relate to this system unless noted otherwise.

2. The coordinate system $\mathbf{K}_{0}^{\mu}$ of the muon. This system is used with the muon polarisation $\mathbf{P}_{\mu}$ as polar axis to describe the decay most naturally. Since $\mathbf{P}_{\mu}$ is precessing in the $(x, y)$ plane with angular frequency $\omega$, its base vectors are time-dependent and are given by

$$
\begin{aligned}
& \hat{\boldsymbol{z}}_{0}^{\mu} \equiv \frac{\mathbf{P}_{\mu}}{\left|\mathbf{P}_{\mu}\right|}=\left(\begin{array}{c}
\cos \omega t \\
\sin \omega t \\
0
\end{array}\right) \\
& \hat{\boldsymbol{x}}_{0}^{\mu} \equiv \hat{\boldsymbol{z}}=\left(\begin{array}{l}
0 \\
0 \\
1
\end{array}\right) \\
& \hat{\boldsymbol{y}}_{0}^{\mu} \equiv \hat{\boldsymbol{z}}_{0} \times \hat{\boldsymbol{x}}_{0}=\left(\begin{array}{c}
\sin \omega t \\
-\cos \omega t \\
0
\end{array}\right) .
\end{aligned}
$$

3. The coordinate system $\mathbf{K}_{0}^{e}$ of the positron. Here the polar axis is given by the momentum of the positron which has been emitted at polar angle $\vartheta_{0}$ and azimuthal angle $\varphi_{0}$ with respect to $\mathbf{K}_{0}^{\mu}$. Its base vectors $\hat{\boldsymbol{x}}_{0}, \hat{\boldsymbol{y}}_{0}$, and $\hat{\boldsymbol{z}}_{0}$, expressed in the muon's coordinate system $\mathbf{K}_{0}^{\mu}$, are:

$$
\hat{\boldsymbol{x}}_{0}=\left(\begin{array}{c}
-\cos \varphi_{0} \cos \vartheta_{0} \\
-\sin \varphi_{0} \cos \vartheta_{0} \\
\sin \vartheta_{0}
\end{array}\right)_{\mathbf{K}_{0}^{\mu}}
$$

$$
\begin{aligned}
& \hat{\boldsymbol{y}}_{0}=\left(\begin{array}{c}
\sin \varphi_{0} \\
-\cos \varphi_{0} \\
0
\end{array}\right)_{\mathbf{K}_{0}^{\mu}} \\
& \hat{\boldsymbol{z}}_{0}=\left(\begin{array}{c}
\cos \varphi_{0} \sin \vartheta_{0} \\
\sin \varphi_{0} \sin \vartheta_{0} \\
\cos \vartheta_{0}
\end{array}\right)_{\mathbf{K}_{0}^{\mu}} .
\end{aligned}
$$

In the global coordinate system $\mathbf{K}$ its base vectors are functions of the time $t$ of the decay and are given by:

$$
\begin{aligned}
& \hat{\boldsymbol{x}}_{0}=\left(\begin{array}{c}
\sin \vartheta_{0} \cos \omega t-\sin \varphi_{0} \cos \vartheta_{0} \sin \omega t \\
\sin \varphi_{0} \cos \vartheta_{0} \cos \omega t+\sin \vartheta_{0} \sin \omega t \\
-\cos \varphi_{0} \cos \vartheta_{0}
\end{array}\right) \\
& \hat{\boldsymbol{y}}_{0}=\left(\begin{array}{c}
-\cos \varphi_{0} \sin \omega t \\
\cos \varphi_{0} \cos \omega t \\
\sin \varphi_{0}
\end{array}\right) \\
& \hat{\boldsymbol{z}}_{0}=\left(\begin{array}{c}
\cos \vartheta_{0} \cos \omega t+\sin \varphi_{0} \sin \vartheta_{0} \sin \omega t \\
-\sin \varphi_{0} \sin \vartheta_{0} \cos \omega t+\cos \vartheta_{0} \sin \omega t \\
\cos \varphi_{0} \sin \vartheta_{0}
\end{array}\right) .
\end{aligned}
$$

The experiment selects positrons emitted close to the symmetry axis $\left(\vartheta_{0}=90^{\circ}, \varphi_{0}=0^{\circ}\right)$ of the apparatus. For positrons emitted exactly into the direction of the 
symmetry axis one obtains:

$$
\begin{aligned}
& \hat{\boldsymbol{x}}_{0}=\left(\begin{array}{c}
\cos \omega t \\
\sin \omega t \\
0
\end{array}\right) \\
& \hat{\boldsymbol{y}}_{0}=\left(\begin{array}{c}
-\sin \omega t \\
\cos \omega t \\
0
\end{array}\right) \\
& \hat{\boldsymbol{z}}_{0}=\left(\begin{array}{l}
0 \\
0 \\
1
\end{array}\right) .
\end{aligned}
$$

The polarisation vector of the $e^{+}$is described most easily in the coordinate system $\mathbf{K}_{0}^{e}$ (see Sect. 2, (13)):

$$
\mathbf{P}_{e^{+}}=P_{\mathrm{T}_{1}}\left(\vartheta_{0}, x\right) \hat{\boldsymbol{x}}_{0}+P_{\mathrm{T}_{2}}\left(\vartheta_{0}, x\right) \hat{\boldsymbol{y}}_{0}+P_{\mathrm{L}} \hat{\boldsymbol{z}}_{0},
$$

where $P_{\mathrm{L}}$ designates the longitudinal positron polarisation. According to (34)-(35) they can be factorised as

$$
\begin{aligned}
P_{\mathrm{T}_{1}}\left(\vartheta_{0}, x\right) & =P_{\mu} P_{1}(x) \sin \vartheta_{0} \\
P_{\mathrm{T}_{2}}\left(\vartheta_{0}, x\right) & =P_{\mu} P_{2}(x) \sin \vartheta_{0} \\
P_{\mathrm{L}} & \equiv P_{3} \approx 1 .
\end{aligned}
$$

The three components $P_{1}, P_{2}$ and $P_{3}$ are functions of the reduced positron energy $x$ and also depend on several muon decay parameters $[11,16]$.

4. The direction of the positron in the global system $\mathbf{K}$ is given by a polar angle $\chi$ and an azimuthal angle $\alpha$. This defines the coordinate system $\mathbf{K}_{\nu}$ with unit vectors $\hat{\boldsymbol{x}}_{\nu}$, $\hat{\boldsymbol{y}}_{\nu}$ and $\hat{\boldsymbol{z}}_{\nu}$ :

$$
\begin{aligned}
& \hat{\boldsymbol{x}}_{\nu}=\frac{1}{\sqrt{R^{2}+T^{2}}}\left(\begin{array}{c}
T \\
0 \\
-R
\end{array}\right) \\
& \hat{\boldsymbol{y}}_{\nu}=\frac{1}{\sqrt{R^{2}+T^{2}}}\left(\begin{array}{c}
-R S \\
R^{2}+T^{2} \\
-S T
\end{array}\right) \\
& \hat{\boldsymbol{z}}_{\nu}=\left(\begin{array}{c}
R \\
S \\
T
\end{array}\right),
\end{aligned}
$$

with the abbreviations

$$
\begin{aligned}
R & \equiv \sin \chi \cos \alpha \\
S & \equiv \sin \chi \sin \alpha \\
T & \equiv \cos \chi .
\end{aligned}
$$

For each detector pair $(i, j)$ with an azimuthal orientation angle $\psi$ the expected annihilation rate will be calculated as a function of the precessing muon polarisation and thereby as a function of time. For the calculation of the annihilation cross section the polarisation vectors $\mathbf{P}$ and $\mathbf{Q}$ of the $e^{+}$and the $e^{-}$, respectively, are needed in the individual coordinate system $\mathbf{K}_{\nu}$ of the $\nu$ th positron as well as in the global system $\mathbf{K}$.
The annihilation cross section of McMaster (see Sect. 3) has been calculated for the c.m. system of the $e^{+} e^{-}$pair in a plane given by the momentum of the positron and the momenta of the annihilation quanta (see Fig. 3). There the momentum of the positron is opposite to the polar axis $\hat{\tilde{\boldsymbol{z}}}$ used. In the following section I transform the annihilation cross section (55) into the global system.

\subsection{Annihilation cross section in the global coordinate system}

In this section the annihilation cross section in the global coordinate system is derived for an ensemble of positrons emitted in different directions. Figure 4 shows the front view as seen in the direction of $\hat{\boldsymbol{z}}$. A $\mu^{+}$decays at $\mathbf{r}_{0}$. The $e^{+}$is emitted under the azimuthal angle $\alpha$ and the polar angle $\chi$ (not shown). The $e^{+}-$trajectory intersects with the BGO front plane at $\mathbf{r}_{1}$. The two annihilation quanta hit the BGO front plane at $\mathbf{r}_{3}$ and $\mathbf{r}_{4}\left(E_{3} \geq E_{4}\right)$. The difference vector $\mathbf{r}_{34}=\mathbf{r}_{3}-\mathbf{r}_{4}$ forms the angle $\psi$ with the $\hat{\boldsymbol{x}}-$ axis $(0 \leq \psi<2 \pi)$.

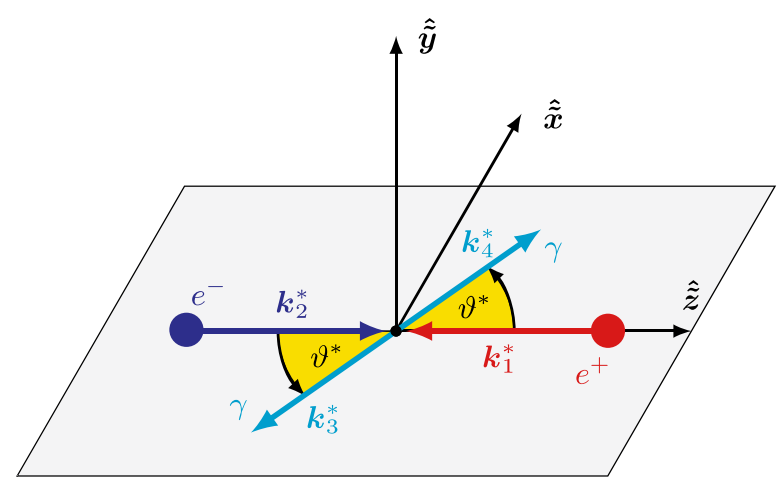

Fig. 3. Center of momentum system for the two-quantum annihilation of positrons with electrons according to McMaster[23]. In this paper I designate the coordinates used by McMaster with a tilde

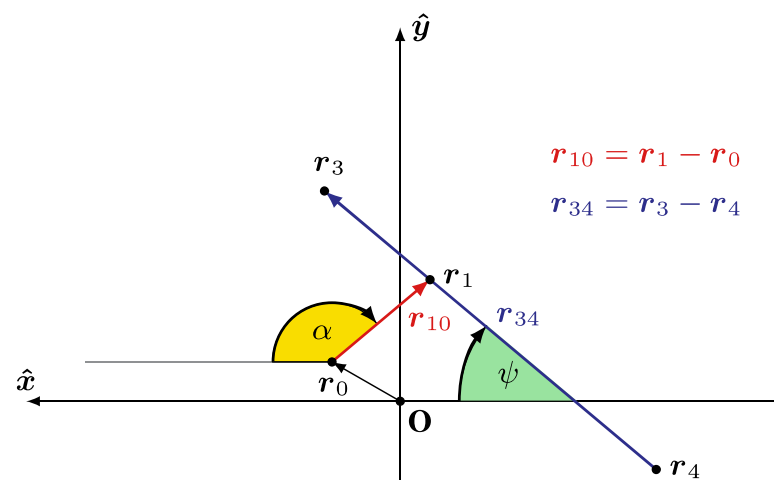

Fig. 4. Front view of the decay of a muon at position $\mathbf{r}_{0}$ and of the subsequent annihilation of the decay positron at position $\mathbf{r}_{1}$ (see text above), as seen in the global coordinate system $\mathbf{K}$. Note that $\alpha$ is the azimuthal angle of emission of the $e^{+}$and therefore different for different decays, while $\psi$ is the azimuthal angle of a given detector pair and therefore constant in time 

to

In the global system the positron polarisation amounts

$$
\mathbf{P}=\left(\begin{array}{l}
P_{x} \\
P_{y} \\
P_{z}
\end{array}\right)
$$

while the electron polarisation is fixed in the positive or negative $x$-direction:

$$
\mathbf{Q}=\left(\begin{array}{c}
Q_{0} \\
0 \\
0
\end{array}\right)
$$

I calculate the annihilation cross section by transforming $\mathbf{P}$ and $\mathbf{Q}$ into the system $\widetilde{\mathbf{K}}$ of McMaster:

$$
\begin{aligned}
& \mathbf{P}=\left(\begin{array}{l}
P_{\mathrm{a}} \\
P_{\mathrm{b}} \\
P_{\mathrm{c}}
\end{array}\right)_{\widetilde{\mathbf{K}}}=\mathbb{M}\left(\begin{array}{c}
P_{x} \\
P_{y} \\
P_{z}
\end{array}\right) \\
& \mathbf{Q}=\left(\begin{array}{l}
Q_{\mathrm{a}} \\
Q_{\mathrm{b}} \\
Q_{\mathrm{c}}
\end{array}\right)_{\widetilde{\mathbf{K}}}=\mathbb{M}\left(\begin{array}{c}
Q_{0} \\
0 \\
0
\end{array}\right) .
\end{aligned}
$$

The polarisation terms in the annihilation cross section (55) are then given by

$$
\begin{aligned}
P_{\mathrm{a}} Q_{\mathrm{a}} & =M_{11} Q_{0}\left(M_{11} P_{x}+M_{12} P_{y}+M_{13} P_{z}\right) \\
P_{\mathrm{b}} Q_{\mathrm{b}} & =M_{21} Q_{0}\left(M_{21} P_{x}+M_{22} P_{y}+M_{23} P_{z}\right) \\
P_{\mathrm{c}} Q_{\mathrm{c}} & =M_{31} Q_{0}\left(M_{31} P_{x}+M_{32} P_{y}+M_{33} P_{z}\right) .
\end{aligned}
$$

The elements of the transformation matrix $\mathbb{M}$, given as column vectors, are:

$$
\begin{aligned}
M_{i 1}= & \left(\begin{array}{c}
-R S U \sin \psi+U\left(S^{2}-T^{2}\right) \cos \psi \\
-T U \sin \psi \\
-R
\end{array}\right) \\
M_{i 2}= & \left(\begin{array}{c}
U\left(R^{2}-T^{2}\right) \sin \psi-R S U \cos \psi \\
T U \cos \psi \\
-S
\end{array}\right) \\
M_{i 3}= & \left(\begin{array}{c}
S T U \sin \psi+R T U \cos \psi \\
R U \sin \psi-S U \cos \psi \\
-T
\end{array}\right) .
\end{aligned}
$$

with the following abbreviations:

$$
\begin{aligned}
& R=\sin \chi \cos \alpha \\
& S=\sin \chi \sin \alpha \\
& T=\cos \chi \\
& U=\frac{1}{\sqrt{T^{2}+(R \sin \psi-S \cos \psi)^{2}}}
\end{aligned}
$$

At this point I substitute the polarisation components $P_{x}$, $P_{y}$, and $P_{z}$ in the global system with the individual polarisation components $P_{1}, P_{2}$ and $P_{3}$, thereby making use of (70)-(72) and (76)-(79). The latter three components contain only the information from the matrix element of the decay, while the angular information is separated. I also make use of the fact that $\chi \ll 1$ and expand both $\mathbb{M}$ and $\mathbf{P}$ up to first order in $\chi$ :

$$
\begin{aligned}
& \mathbb{M}=\left(\begin{array}{ccc}
-\cos \psi & -\sin \psi & \chi \cos (\psi-\alpha) \\
-\sin \psi & \cos \psi & \chi \sin (\psi-\alpha) \\
-\chi \cos \alpha & -\chi \sin \alpha & -1
\end{array}\right) \\
& \mathbf{P}=\left(\begin{array}{c}
P_{1} \cos \omega t-P_{2} \sin \omega t+\chi P_{3} \cos \alpha \\
P_{1} \sin \omega t+P_{2} \cos \omega t+\chi P_{3} \sin \alpha \\
-\chi P_{1} \cos (\omega t-\alpha)+\chi P_{2} \sin (\omega t-\alpha)+P_{3}
\end{array}\right) .
\end{aligned}
$$

The polarisation terms are then:

$$
\begin{aligned}
& P_{a} Q_{a}=Q_{0} P_{\mu} \cos \psi\left\{P_{1} \cos (\omega t-\psi)-P_{2} \sin (\omega t-\psi)\right\} \\
& P_{b} Q_{b}=Q_{0} P_{\mu} \sin \psi\left\{-P_{1} \sin (\omega t-\psi)-P_{2} \cos (\omega t-\psi)\right\} \\
& P_{c} Q_{c}=Q_{0} P_{3} \chi \cos \alpha .
\end{aligned}
$$

The cross section, finally, in this approximation is given by:

$$
\begin{aligned}
\frac{\mathrm{d} \sigma}{\mathrm{d} \Omega}= & \sigma_{0}+\sigma_{x} P_{\mu} Q_{0} \cos \psi\left[P_{1} \cos (\omega t-\psi)-P_{2} \sin (\omega t-\psi)\right] \\
& +\sigma_{y} P_{\mu} Q_{0} \sin \psi\left[-P_{1} \sin (\omega t-\psi)-P_{2} \cos (\omega t-\psi)\right] \\
& +\sigma_{z} Q_{0}\left[P_{3} \chi \cos \alpha\right] .
\end{aligned}
$$

The behaviour of this result is illustrated in Fig. 5 for completely transversely polarised positrons $\left(P_{\mathrm{T}_{1}}=1\right)$ and electrons $(Q=1)$ (which implies $P_{\mu}=1$ ) with $E_{3}=E_{4}=50 \mathrm{~m}$

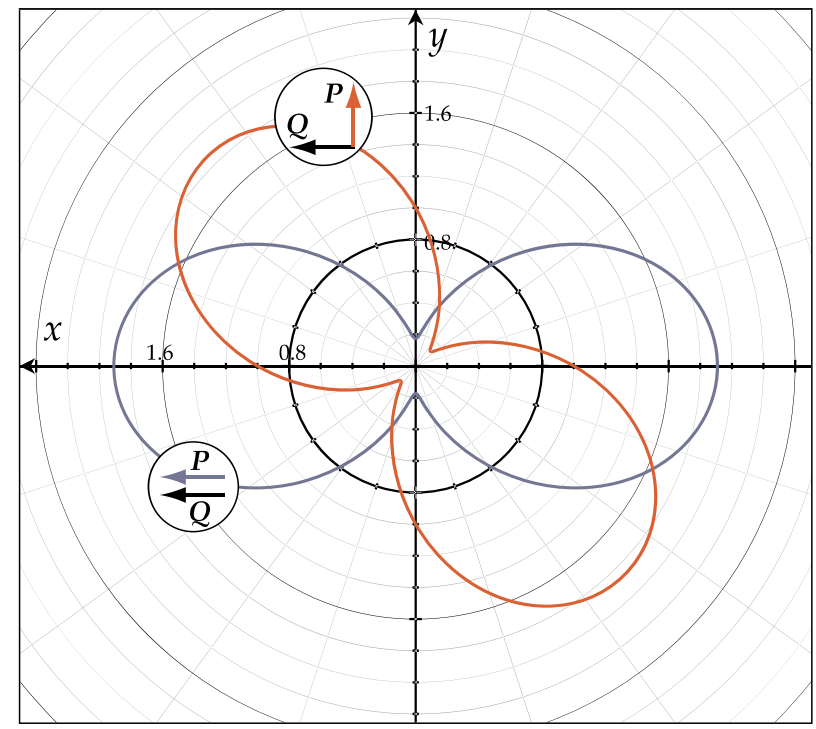

Fig. 5. Intensity distributions of the annihilation quanta at $E_{3}=E_{4}=50 m_{e}$ for parallel spins $\left(e^{-}: \mathbf{Q}=1, e^{+}: \mathbf{P}_{\mathrm{T}}=1\right)$ and for perpendicular spins. The maximum of the intensity lies on the bisector of the angle $\omega t$ between the two spins. Thus the "figure of eight" moves with angular frequency $\omega / 2$. For a fixed detector pair at azimuthal angle $\psi$ the time dependence is still given by the angular frequency $\omega$ due to the two symmetric lobes of the "figure of eight" 
There the normalized annihilation rate is displayed in function of the azimuthal angle $\psi$. At time $t=0, \mathbf{P}_{\mathrm{T}} \| \mathbf{Q}$, and the intensity distribution of the annihilation quanta has the shape of a horizontally lying "figure of eight" in the polar diagram. At $t=\pi /(2 \omega), \mathbf{P}_{\mathrm{T}} \perp \mathbf{Q}$, and the "figure of eight" has moved by $\pi / 4$. The maximum of the intensity lies on the bisector of the angle $\omega t$ between the two spins. Thus the "figure of eight" moves with angular frequency $\omega / 2$. For a fixed detector pair at azimuthal angle $\psi$ the time dependence is still given by the angular frequency $\omega$ due to the two symmetric lobes of the "figure of eight".
This is due to the fact that the cross section depends on time by a harmonic with angular frequency $\omega$, but on the azimuthal orientation angle $\psi$ of the two annihilation quanta by a harmonic of $2 \psi$. By transforming the electron and the positron polarisation into the system of McMaster which is oriented at the azimuthal angle $\psi$ terms with $\cos \psi$ and $\sin \psi$ appear in second order. In contrast to that the time dependence of the positron polarisation is due to the precession of the muon polarisation and thus does not effect the electron polarisation. Therefore terms with $\cos \omega t$ and $\sin \omega t$ enter (105) only linearly.
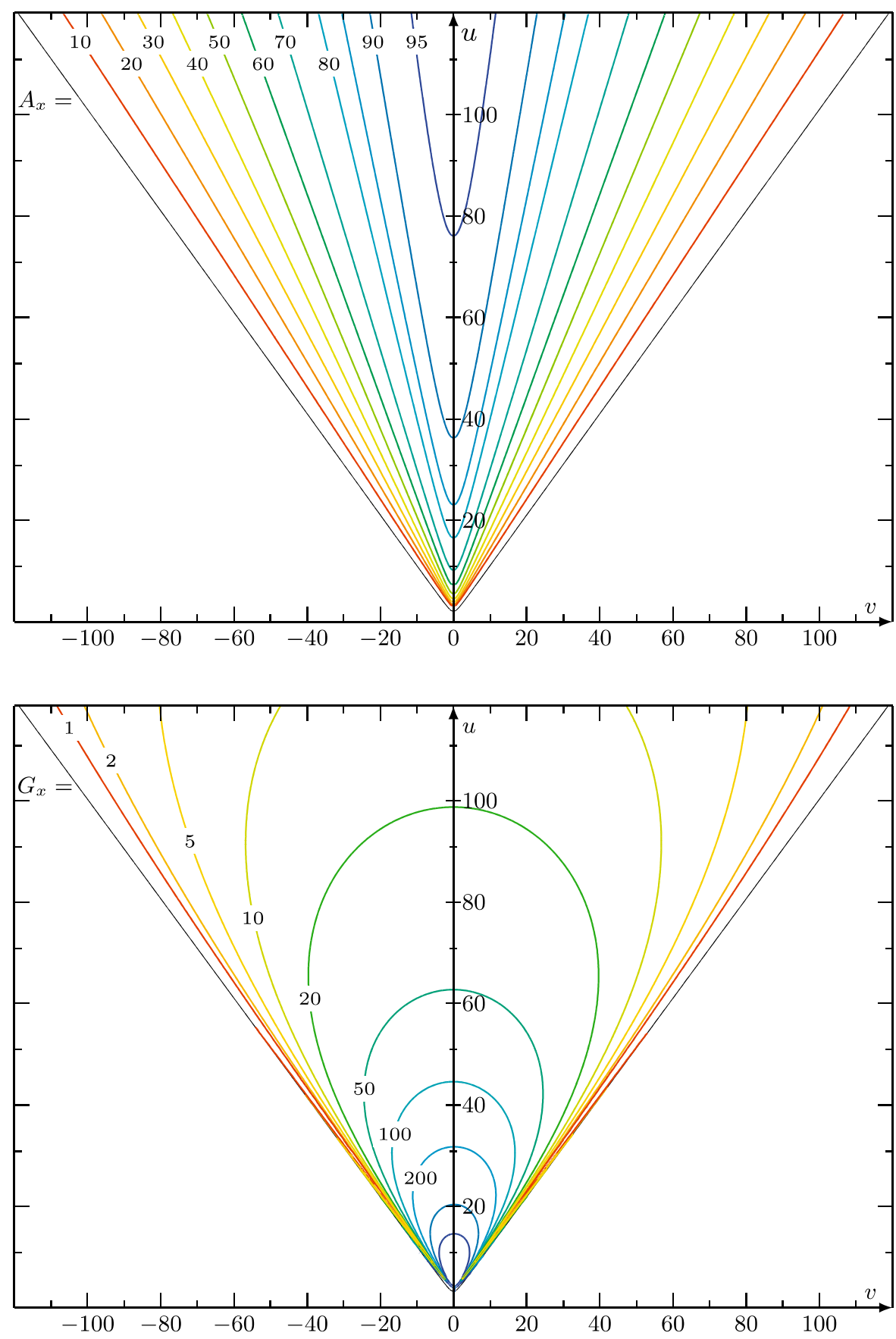

Fig. 6. Contour lines for the transverse analysing power $A_{x}$ (in \%) as a function of the sum $u=\left(E_{3}+E_{4}\right) / m_{e}$ and the difference $v=\left(E_{3}-E_{4}\right) / m_{e}$ of the normalized photon energies $E_{3}$ and $E_{4}$. The outmost line is the kinematic boundary
Fig. 7. Contour lines for the Figure of Merit $G_{x}$ (arbitrary units, equal for all $\left.G_{j}, j=x, y, z\right)$ as a function of the sum $u=\left(E_{3}+E_{4}\right) / m_{e}$ and the difference $v=$ $\left(E_{3}-E_{4}\right) / m_{e}$ of the normalized photon energies $E_{3}$ and $E_{4}$. The outmost line is the kinematic boundary 


\subsection{Time dependent annihilation rate}

The normalized time distribution is then given by:

$$
\begin{aligned}
\frac{1}{\sigma_{0}} \frac{\mathrm{d} \sigma}{\mathrm{d} \Omega}= & 1+P_{\mu} Q \cos \omega t\left(P_{1} G+P_{2} H\right) \\
& +P_{\mu} Q \sin \omega t\left(P_{1} H-P_{2} G\right) \\
& +Q P_{3} A_{z} \chi \cos \alpha,
\end{aligned}
$$

with

$$
\begin{aligned}
G & =\left(A_{x} \cos ^{2} \psi+A_{y} \sin ^{2} \psi\right) \\
H & =\sin \psi \cos \psi\left(A_{x}-A_{y}\right)
\end{aligned}
$$

$$
\begin{aligned}
& A_{x} \equiv \frac{\sigma_{x}}{\sigma_{0}}=\frac{A+B-C-D}{A+B+C+D} \\
& A_{y} \equiv \frac{\sigma_{y}}{\sigma_{0}}=\frac{A-B+C-D}{A+B+C+D} \\
& A_{z} \equiv \frac{\sigma_{z}}{\sigma_{0}}=\frac{-A+B+C-D}{A+B+C+D} .
\end{aligned}
$$

By summing over annihilation events for a given angle $\psi$, but distributed symmetrically with respect to the azimuthal angle $\alpha$ of the positron, the spatial dependence of the distribution (106) on the longitudinal positron polarisation component $P_{3}$ vanishes. The remaining time distri-
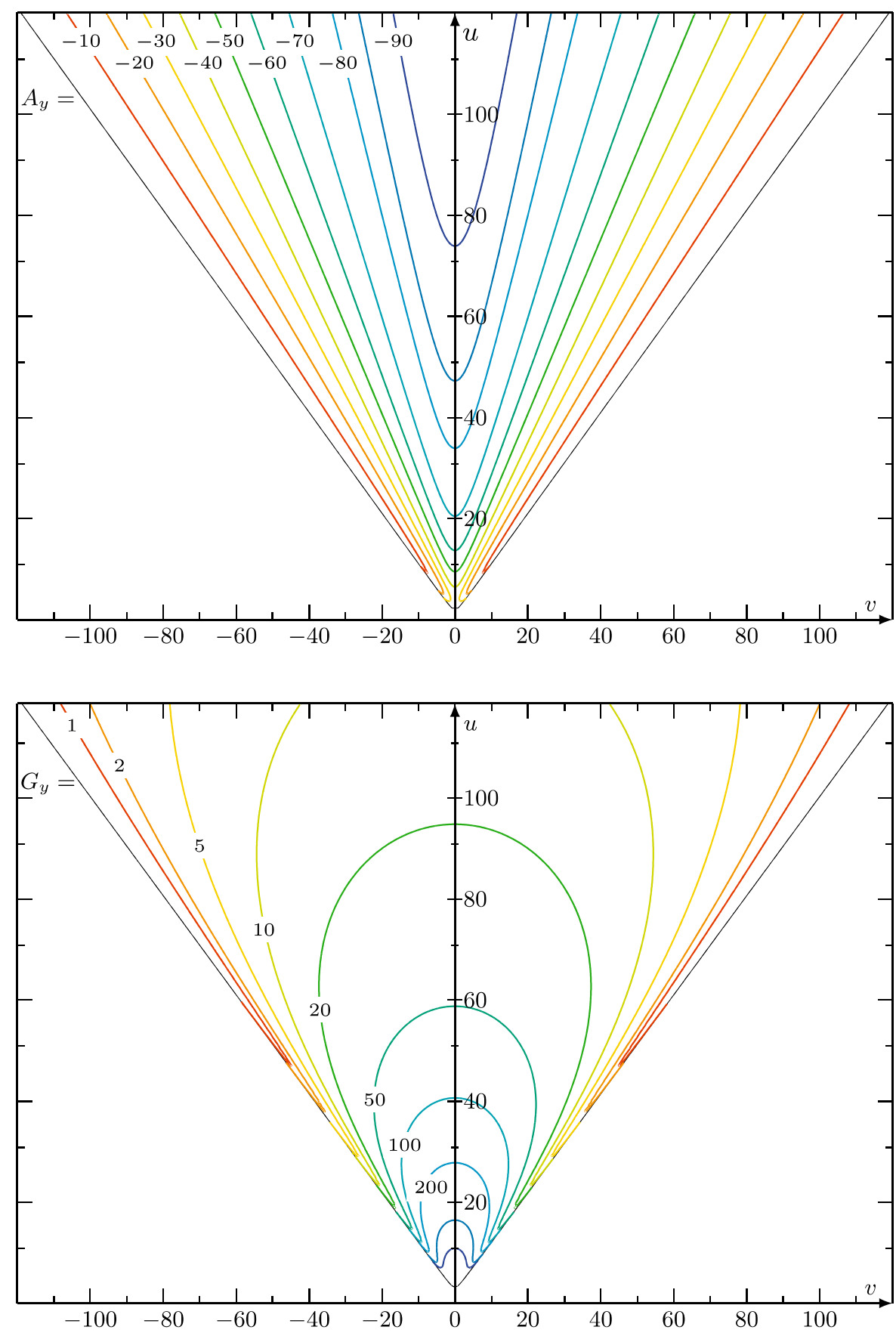

Fig. 8. Contour lines for the transverse analysing power $A_{y}$ (in \%) as a function of the sum $u=\left(E_{3}+E_{4}\right) / m_{e}$ and the difference $v=\left(E_{3}-E_{4}\right) / m_{e}$ of the normalized photon energies $E_{3}$ and $E_{4}$. The outmost line is the kinematic boundary
Fig. 9. Contour lines for the Figure of Merit $G_{y}$ (arbitrary units, equal for all $\left.G_{j}, j=x, y, z\right)$ as a function of the sum $u=\left(E_{3}+E_{4}\right) / m_{e}$ and the difference $v=$ $\left(E_{3}-E_{4}\right) / m_{e}$ of the normalized photon energies $E_{3}$ and $E_{4}$. The outmost line is the kinematic boundary 
bution is:

$$
\begin{aligned}
\frac{1}{\sigma_{0}} \frac{\mathrm{d} \sigma}{\mathrm{d} \Omega}= & 1+P_{\mu} Q \cos \omega t\left(P_{1} G+P_{2} H\right) \\
& +P_{\mu} Q \sin \omega t\left(P_{1} H-P_{2} G\right) .
\end{aligned}
$$

The quantities $G$ and $H$ are the effective analysing powers. In the experiment of Burkard et al. $[17,26]$ they were calculated for the four angles $\psi=0^{\circ}, 45^{\circ}, 90^{\circ}$ and $135^{\circ}$, corresponding to the four different possible orientations of the detector pairs for the four NaI detectors used there. With the 127 BGO detectors of this experiment representing a continuous surface for the detection of the annihila- tion quanta, the full angular dependence of the analysing powers as given by (107) and (108) has been derived to get the best possible sensitivity for the transverse polarisation. They are explicitly functions of $\psi$ and implicitly of the c.m. energy of the $e^{+} e^{-}$system and of the polar emission angle of the photons in the c.m. system. For the analysis of the data it is more convenient to make use of the measured photon energies $E_{3}$ and $E_{4}$ in the following way:

$$
\begin{aligned}
H & \equiv H(\psi, u, v) \\
G & \equiv G(\psi, u, v),
\end{aligned}
$$
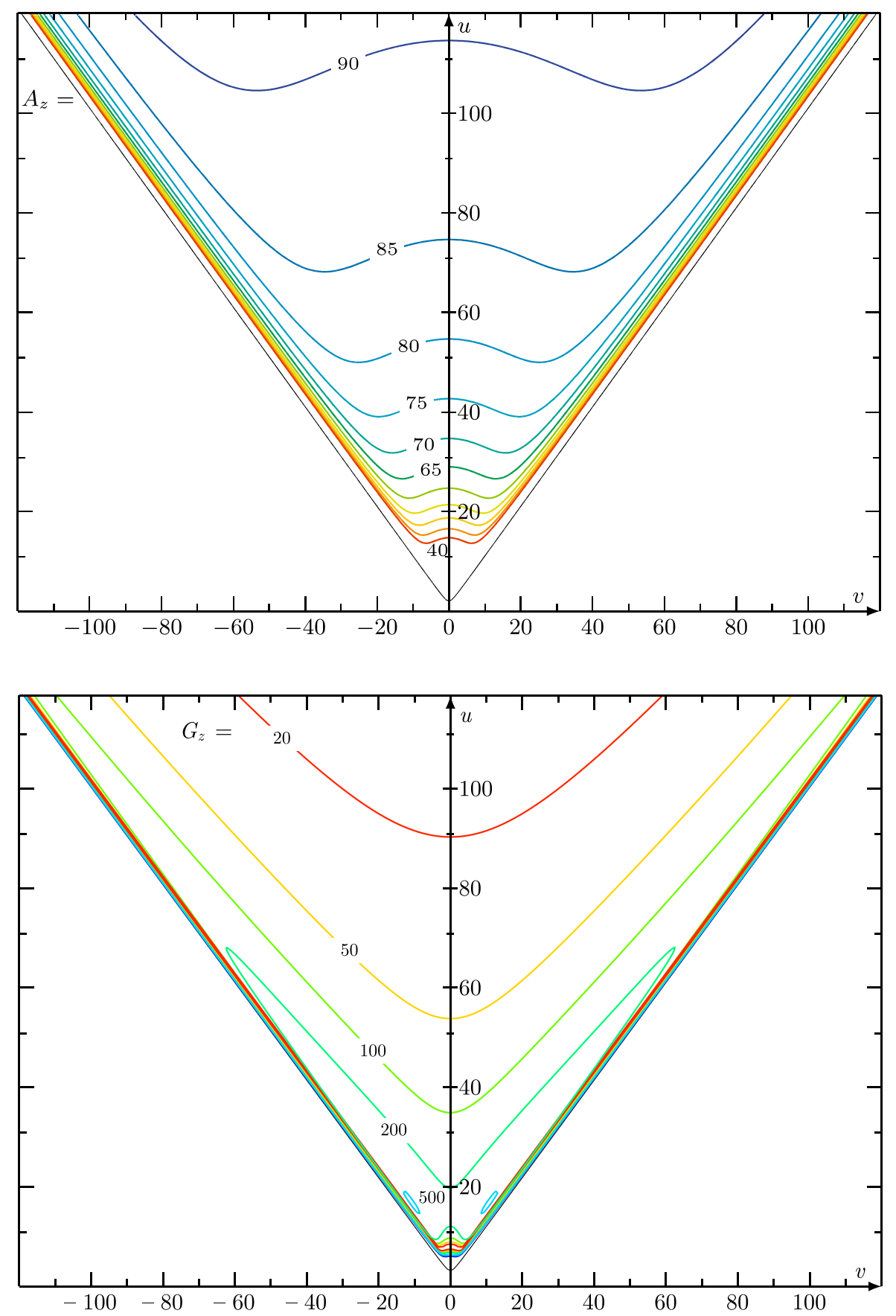

Fig. 10. Contour lines for the longitudinal analysing power $A_{z}$ (in \%) as a function of the sum $u=\left(E_{3}+E_{4}\right) / m_{e}$ and the difference $v=\left(E_{3}-E_{4}\right) / m_{e}$ of the normalized photon energies $E_{3}$ and $E_{4}$. The outmost line is the kinematic boundary

Fig. 11. Contour lines for the Figure of Merit $G_{z}$ (arbitrary units, equal for all $\left.G_{j}, j=x, y, z\right)$ as a function of the sum $u=\left(E_{3}+E_{4}\right) / m_{e}$ and the difference $v=$ $\left(E_{3}-E_{4}\right) / m_{e}$ of the normalized photon energies $E_{3}$ and $E_{4}$. The outmost line is the kinematic boundary 
with

$$
\begin{aligned}
& u=\left(E_{3}+E_{4}\right) / m_{e} \\
& v=\left(E_{3}-E_{4}\right) / m_{e} .
\end{aligned}
$$

Note that due to energy loss of the positron $E_{3}+E_{4}=$ $E_{1}^{\prime}<m+E_{1}$, where $E_{1}$ is the positron initial energy.

The analysing powers $A_{x}$ and their corresponding Figures of Merit in the lab system $G_{x}, A_{y}$ and $G_{y}$ as well as $A_{z}$ and $G_{z}$ are displayed as contour lines in Figs. 6-11, respectively. Their absolute values $\left|A_{j}\right|$ are remarkably large in much of the kinematically allowed region. In Appendix A these three analysing powers and their corresponding Figures of Merit are given.

\subsection{Measurement of the longitudinal polarisation}

The longitudinal polarization can be determined according to (55). One needs, however, to turn the magnetised analyser foil by an angle (usually $45^{\circ}$ ) in order to get a component of the $e^{-}$polarisation parallel to $P_{\mathrm{L}}[21,24]$. To restore the symmetry of the experiment one measures alternatingly with the foil once turned to the right and then to the left. This procedure has to be controlled carefully in order not to introduce systematic errors. A possible alternative is a measurement with the setup used for the measurement of the transverse polarization, where the analysing foil is perpendicular to the average positron direction of flight. In this case one should use unpolarised muons or average over the time-dependent parts of (105). One then gets a small signal proportional to $\chi$. A measurement of the rate asymmetry, obtained from measurements with positive and with negative electron polarisation, in the range $80^{\circ} \leq \chi \leq 100^{\circ}$, for example, will show a linear dependence on $\chi$ with a change of sign at $\chi=90^{\circ}$. The disadvantage of the smaller signal could be outweighed by the gain of symmetry of this setup.

\section{Appendix A: Analysing powers and figures of Merit}

\section{A.1 Figures of Merit $G_{j}$}

In the c.m.system, the figure of Merit (FoM) $G_{j}(j=$ $x, y, z)$ is defined as:

$$
\begin{aligned}
G_{j}^{*} & :=\frac{\mathrm{d} \sigma_{0}}{\mathrm{~d} \cos \vartheta^{*}}\left(\frac{\mathrm{d} \sigma_{j} / \mathrm{d} \cos \vartheta^{*}}{\mathrm{~d} \sigma_{0} / \mathrm{d} \cos \vartheta^{*}}\right)^{2} \\
& =\frac{\left(\mathrm{d} \sigma_{j} / \mathrm{d} \cos \vartheta^{*}\right)^{2}}{\mathrm{~d} \sigma_{0} / \mathrm{d} \cos \vartheta^{*}} \quad(j=x, y, z) \\
& =A_{j}^{2} \frac{A+B+C+D}{\beta^{2} \gamma^{2}\left(1-\beta^{2} \cos ^{2} \vartheta^{*}\right)^{2}} \\
& =A_{j}^{2} \frac{2(A+B+C+D)}{(u-2)\left(1-\frac{v^{2}}{u^{2}}\right)^{2}} .
\end{aligned}
$$

With the laboratory energy $u$ given, the free parameter is the energy difference $v$ :

$$
\begin{aligned}
v & =\sqrt{u(u-2)} \cos \vartheta^{*} \\
\Rightarrow \mathrm{d} v & =\sqrt{u(u-2)} \mathrm{d} \cos \vartheta^{*} .
\end{aligned}
$$

The cross section in the lab system is therefore:

$$
\begin{aligned}
\frac{\mathrm{d} \sigma_{0}}{\mathrm{~d} v} & =\frac{\mathrm{d} \sigma_{0}}{\mathrm{~d} \cos \vartheta^{*}} \frac{\mathrm{d} \cos \vartheta^{*}}{\mathrm{~d} v} \\
& =\frac{\mathrm{d} \sigma_{0}}{\mathrm{~d} \cos \vartheta^{*}} \frac{1}{\sqrt{u(u-2)}} .
\end{aligned}
$$

The FoM $G_{j}$ in the lab system is then:

$$
\begin{aligned}
G_{j} & =\frac{\mathrm{d} \sigma_{0}}{\mathrm{~d} \cos \vartheta^{*}}\left(\frac{\mathrm{d} \sigma_{j} / \mathrm{d} \cos \vartheta^{*}}{\mathrm{~d} \sigma_{0} / \mathrm{d} \cos \vartheta^{*}}\right)^{2} \frac{1}{\sqrt{u(u-2)}} \\
& =A_{j}^{2} \frac{2(A+B+C+D)}{u^{1 / 2}(u-2)^{3 / 2}\left(1-\frac{v^{2}}{u^{2}}\right)^{2}} .
\end{aligned}
$$

\section{A.2 Analysing power $A_{x}$ and figure of Merit $G_{x}$}

$$
\begin{gathered}
\begin{aligned}
& A_{x}=\frac{(A+B)^{\prime}-(C+D)^{\prime}}{(A+B)^{\prime}+(C+D)^{\prime}} \\
& G_{x}=2 \frac{\frac{\left[(A+B)^{\prime}-(C+D)^{\prime}\right]^{2}}{(A+B)^{\prime}+(C+D)^{\prime}}}{u^{9 / 2}(u-2)^{5 / 2}\left(1-\frac{v^{2}}{u^{2}}\right)^{2}} \\
& \text { with } \quad(A+B)^{\prime}:=u^{4}(u-2)(A+B) \\
&=2 v^{4}-v^{2} u(u-2)(u+4)+u^{2}(u-2)^{2}(u+4) \\
&(C+D)^{\prime}:=u^{4}(u-2)(C+D) \\
&=-u v^{4}+v^{2} u^{2}(u-2)+2 u^{3}(u-2)
\end{aligned}
\end{gathered}
$$

\section{A.3 Analysing power $A_{y}$ and figure of Merit $G_{y}$}

$$
\begin{gathered}
A_{y}=\frac{(A+C)^{\prime}-(B+D)^{\prime}}{(A+C)^{\prime}+(B+D)^{\prime}} \\
G_{y}=2 \frac{\frac{\left[(A+C)^{\prime}-(B+D)^{\prime}\right]^{2}}{(A+C)^{\prime}+(B+D)^{\prime}}}{u^{9 / 2}(u-2)^{3 / 2}\left(1-\frac{v^{2}}{u^{2}}\right)^{2}} \\
\text { with } \quad(A+C)^{\prime}:=u^{4}(A+C) \\
=-v^{4}+v^{2} u(u-4)+4 u^{2}(u-2) \\
(B+D)^{\prime}:=u^{4}(C+D) \\
=-v^{2} u^{2}+u^{4}
\end{gathered}
$$




\section{A.4 Analysing power $A_{z}$ and figure of Merit $G_{z}$}

$$
\begin{aligned}
A_{z} & =\frac{(B+C)^{\prime}-(A+D)^{\prime}}{(B+C)^{\prime}+(A+D)^{\prime}} \\
G_{z} & =2 \frac{\frac{\left[(B+C)^{\prime}-(A+D)^{\prime}\right]^{2}}{(B+C)^{\prime}+(A+D)^{\prime}}}{u^{9 / 2}(u-2)^{5 / 2}\left(1-\frac{v^{2}}{u^{2}}\right)^{2}} \\
& \operatorname{with}(B+C)^{\prime}:=u^{4}(u-2)(B+C) \\
= & -v^{4} u+u^{3}(u-2)^{2} \\
(A+D)^{\prime} & :=u^{4}(u-2)(A+D) \\
& =2 v^{4}-4 v^{2} u(u-2)+2 u^{2}(u-2)(3 u-4)
\end{aligned}
$$

\section{Appendix B: Simulation of muon decay}

\section{B.1 Probability density function}

Given the two-dimensional normalised decay probability (see the denominator of (18) to (20))

$$
\begin{aligned}
g(x, y)=\frac{\mathrm{d}^{2} \Gamma}{\mathrm{d} x \mathrm{~d} y} & =F_{\mathrm{IS}}(x)+P_{\mu} \cos \vartheta F_{\mathrm{AS}}(x) \\
& :=f_{1}(x)+P_{\mu} y f_{2}(x),
\end{aligned}
$$

with (see (14))

$$
\begin{aligned}
x & =\frac{E_{e}}{W_{\mu e}} \\
y & =\cos \vartheta \\
f_{1}(x) & =-2 x^{3}+3 x^{2} \\
f_{2}(x) & =+2 x^{3}-x^{2} .
\end{aligned}
$$

In (B.2) standard $V-A$ values have been assumed and terms proportional to the electron mass have been neglected.

\section{B.2 Density functions of $x$ and $y$}

The marginal probability density function (p.d.f.) of $x$ is

$$
g_{\xi}(x)=\frac{\int_{-1}^{+1} g(x, y) \mathrm{d} y}{\int_{0}^{+1} \mathrm{~d} x \int_{-1}^{+1} g(x, y) \mathrm{d} y}=2 f_{1}(x) .
$$

The conditional density function of $y$ is obtained for a given value $x=x_{\mu}$ :

$$
\begin{aligned}
g_{\eta}\left(y \mid x_{\mu}\right) & =\frac{g\left(x_{\mu}, y\right)}{g_{\xi}\left(x_{\mu}\right)} \\
& =\frac{1}{2}\left(1+y \frac{f_{2}\left(x_{\mu}\right)}{f_{1}\left(x_{\mu}\right)} P_{\mu}\right) .
\end{aligned}
$$

The two density functions are thus:

$$
\begin{aligned}
& g_{\xi}(x)=2 f_{1}(x) \\
& g_{\eta}\left(y \mid x_{\mu}\right)=\frac{1}{2}\left(1+\alpha_{\mu} y\right) \\
& \text { with } \quad \alpha_{\mu}=P_{\mu} \frac{f_{2}\left(x_{\mu}\right)}{f_{1}\left(x_{\mu}\right)} .
\end{aligned}
$$

\section{B.3 Cumulative distribution function of $x$}

$$
\begin{aligned}
2 \int_{0}^{x} f_{1}\left(x^{\prime}\right) \mathrm{d} x^{\prime} & =-x^{4}+2 x^{3} \\
& :=R_{\mu}^{x},
\end{aligned}
$$

with the uniformly distributed random number

$$
R_{\mu}^{x} \in[0,1] .
$$

This equation can be solved numerically with Newton's method. There is a problem at $x=0$, however, where the derivatives of the polynomial are equal to zero. With a threshold energy $x_{t}>0$ we can neglect this problem.

This reduces the range for $R_{\mu}^{x}$ to

$$
\begin{gathered}
1 \geqq R_{\mu}^{x} \geqq-x_{t}^{4}+2 x_{t}^{3} \\
\Rightarrow \quad \alpha_{\mu}=P_{\mu} \frac{2 x_{\mu}-1}{3-2 x_{\mu}} .
\end{gathered}
$$

\section{B.4 Cumulative distribution function of $y$}

One obtains accordingly the conditional cumulative distribution function of $y$ for the value $x_{\mu}$ resp. $\alpha_{\mu}$, as obtained from the random variable $R_{\mu}^{x}$, by generating the uniformly distributed random variable $R_{\mu \nu}^{y}$ with the range

$$
R_{\mu \nu}^{y} \in[0,1] \text {. }
$$

One obtains

$$
\begin{aligned}
R_{\mu \nu}^{y} & :=\frac{1}{2} \int_{-1}^{y}\left(1+\alpha_{\mu} y^{\prime}\right) \mathrm{d} y^{\prime} \\
& =\frac{1}{2}\left[y^{\prime}+\frac{\alpha_{\mu}}{2} y^{\prime}\right]_{-1}^{y} \\
& =\frac{1}{2}\left\{(y+1)+\frac{\alpha_{\mu}}{2}\left(y^{2}-1\right)\right\}
\end{aligned}
$$


The solutions of this quadratic equation are

$$
y_{\mu \nu}=\frac{1}{\alpha_{\mu}}\left\{-1(\stackrel{+}{-}) \sqrt{\left(1-\alpha_{\mu}\right)^{2}+4 \alpha_{\mu} R_{\mu \nu}^{y}}\right\},
$$

whereby only the upper sign constitutes a physical solution. This expression is not determined for $\alpha_{\mu}=0$. This problem can be repaired by transforming it into the following expression:

$$
\begin{aligned}
y_{\mu \nu}= & -\frac{1}{\alpha_{\mu}}\left\{1-\sqrt{\left(1-\alpha_{\mu}\right)^{2}+4 \alpha_{\mu} R_{\mu \nu}^{y}}\right\} \\
& \times \frac{\left(1+\sqrt{\left(1-\alpha_{\mu}\right)^{2}+4 \alpha_{\mu} R_{\mu \nu}^{y}}\right)}{\left(1+\sqrt{\left(1-\alpha_{\mu}\right)^{2}+4 \alpha_{\mu} R_{\mu \nu}^{y}}\right)} \\
\Rightarrow y_{\mu \nu}= & \frac{4 R_{\mu \nu}^{y}-2+\alpha_{\mu}}{1+\sqrt{\left(1-\alpha_{\mu}\right)^{2}+4 \alpha_{\mu} R_{\mu \nu}^{y}}} .
\end{aligned}
$$

\section{Appendix C: Simulation of the polarisation dependent annihilation}

\section{C.1 Differential cross section}

The simulation of the differential annihilation cross section for arbitary electron and positron polarisation is determined according to (55) in the system of McMaster. Figure 12 shows the geometry. The annihilation takes place in the $\hat{\tilde{\boldsymbol{x}}} \hat{\tilde{\boldsymbol{z}}}$ plane.

The system $\mathbf{K}_{\nu}$ has $\hat{\boldsymbol{z}}_{\nu}=-\hat{\tilde{\boldsymbol{z}}}$, with the azimuthal angle $\varphi$ between $\hat{\boldsymbol{x}}_{\nu}$ and $\hat{\tilde{\boldsymbol{x}}}$ (see Fig. 13). The polarisation vectors $\mathbf{P}=\left(P_{\mathrm{c}}, P_{\mathrm{a}}, P_{\mathrm{b}}\right)$ of the $e^{+}$and $\mathbf{Q}=\left(Q_{\mathrm{c}}, Q_{\mathrm{a}}, Q_{\mathrm{b}}\right)$ of the $e^{-}$ $((64)$ and $(65))$ used there are replaced by the components used in the system $\mathbf{K}_{\nu}$ of the positron:

$$
\left(\begin{array}{l}
P_{\mathrm{a}} \\
P_{\mathrm{b}} \\
P_{\mathrm{c}}
\end{array}\right)_{\tilde{\mathbf{K}}}=\left(\begin{array}{ccc}
\cos \varphi & \sin \varphi & 0 \\
\sin \varphi-\cos \varphi & 0 \\
0 & 0 & -1
\end{array}\right)\left(\begin{array}{l}
P_{n 1} \\
P_{n 2} \\
P_{n 3}
\end{array}\right)_{\mathbf{K}_{\nu}}
$$

The polarisation $\mathbf{Q}$ of the electron is transformed accordingly.

We finally obtain:

$$
\begin{aligned}
P_{\mathrm{a}} Q_{\mathrm{a}} & =\frac{1}{2}\left\{X_{\mathrm{T}}+X_{\mathrm{C}} \cos 2 \varphi+X_{\mathrm{S}} \sin 2 \varphi\right\} \\
P_{\mathrm{b}} Q_{\mathrm{b}} & =\frac{1}{2}\left\{X_{\mathrm{T}}-X_{\mathrm{C}} \cos 2 \varphi-X_{\mathrm{S}} \sin 2 \varphi\right\} \\
P_{\mathrm{c}} Q_{\mathrm{c}} & =X_{\mathrm{L}}
\end{aligned}
$$

with

$$
\begin{aligned}
X_{\mathrm{T}} & \equiv P_{n 1} Q_{n 1}+P_{n 2} Q_{n 2} \\
X_{\mathrm{S}} & \equiv P_{n 1} Q_{n 2}+P_{n 2} Q_{n 1} \\
X_{\mathrm{C}} & \equiv P_{n 1} Q_{n 1}-P_{n 2} Q_{n 2} \\
X_{\mathrm{L}} & \equiv P_{n 3} Q_{n 3} .
\end{aligned}
$$
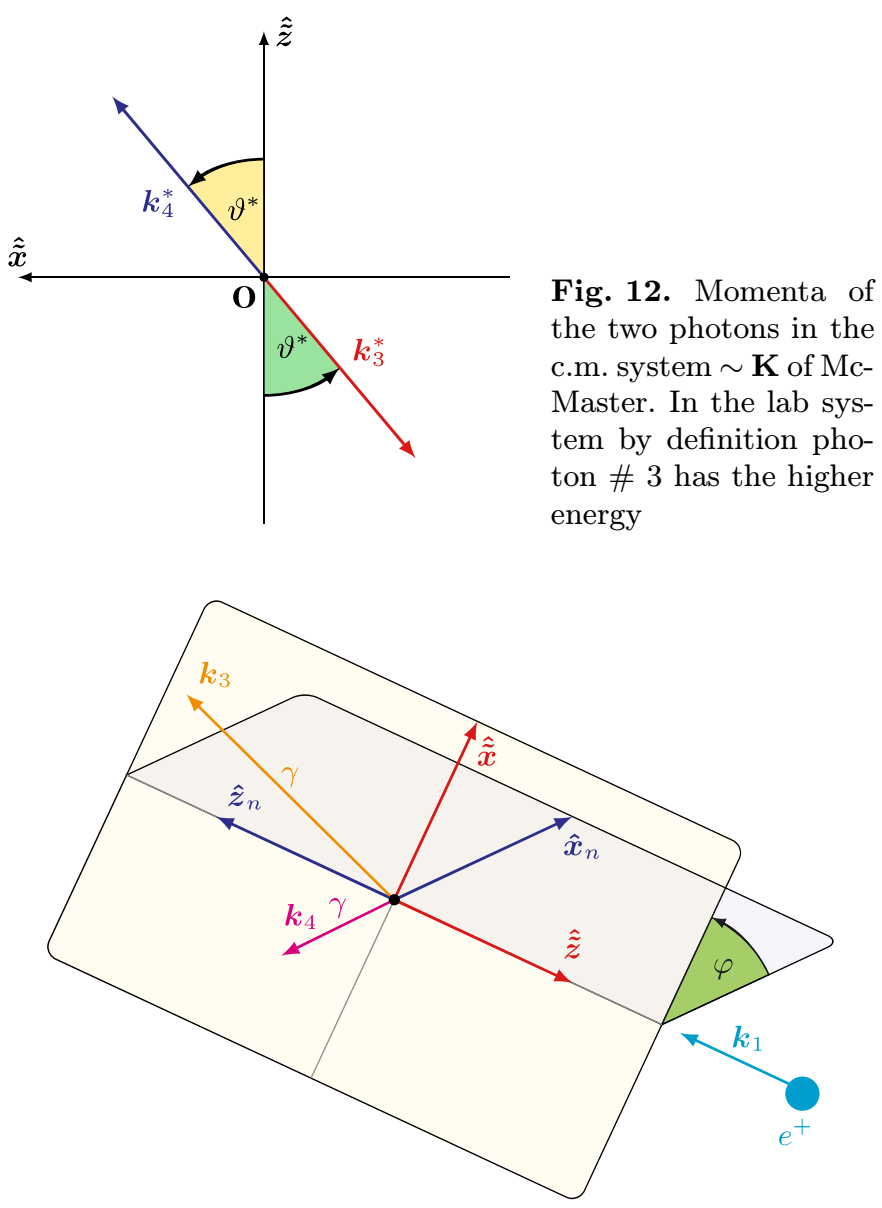

Fig. 13. Plane of annihilation (axes $\hat{\tilde{\boldsymbol{x}}}$ and $\hat{\tilde{\boldsymbol{z}}}$ ) and $\hat{\boldsymbol{x}}_{n}-\hat{\boldsymbol{y}}_{n}$-plane of the positron $\mathbf{K}_{n}$. The positron momentum $\mathbf{k}_{1}$ is along $\hat{\boldsymbol{y}}_{n}$ axis. The $\hat{\tilde{\boldsymbol{x}}}$ axis of the annihilation plane forms the angle $\varphi$ with $\hat{\boldsymbol{x}}_{n}$. By construction, $\hat{\boldsymbol{x}}_{n}$ always lies in a horizontal plane. The momentum vectors of the annihilation quanta in the lab system are designated as $\mathbf{k}_{3}$ and $\mathbf{k}_{4}$

The differential cross section thus becomes:

$$
\begin{aligned}
\frac{\mathrm{d} \sigma}{\mathrm{d} \Omega}= & \sigma_{0}+X_{\mathrm{L}} \sigma_{z}+X_{\mathrm{T}} \frac{\left(\sigma_{x}+\sigma_{y}\right)}{2} \\
& +X_{\mathrm{C}} \frac{\left(\sigma_{x}-\sigma_{y}\right)}{2} \cos 2 \varphi+X_{\mathrm{S}} \frac{\left(\sigma_{x}-\sigma_{y}\right)}{2} \sin 2 \varphi
\end{aligned}
$$

or, in in a more compact form:

$$
\frac{\mathrm{d} \sigma}{\mathrm{d} \Omega}=f_{1}\left(\cos \vartheta^{*}\right)+f_{2}\left(\cos \vartheta^{*}\right) \cos 2 \varphi+f_{3}\left(\cos \vartheta^{*}\right) \sin 2 \varphi,
$$

with

$$
\begin{aligned}
& f_{1}\left(\cos \vartheta^{*}\right)=\sigma_{0}+X_{\mathrm{L}} \sigma_{z}+X_{\mathrm{T}} \frac{\left(\sigma_{x}+\sigma_{y}\right)}{2} \\
& f_{2}\left(\cos \vartheta^{*}\right)=X_{\mathrm{C}} \frac{\left(\sigma_{x}-\sigma_{y}\right)}{2} \\
& f_{3}\left(\cos \vartheta^{*}\right)=X_{\mathrm{S}} \frac{\left(\sigma_{x}-\sigma_{y}\right)}{2} .
\end{aligned}
$$


The functions $f_{i}$, expressed through the terms $A, B, C$ und $D$, are:

$$
\begin{aligned}
f_{1}\left(\cos \vartheta^{*}\right)= & \frac{r_{0}^{2}}{4 \beta \gamma^{2}\left(1-\beta^{2} \cos \vartheta^{*}\right)^{2}}\{[A+B+C+D] \\
& \left.+X_{\mathrm{L}}[-A+B+C-D]+X_{\mathrm{T}}[A-D]\right\} \\
f_{2}\left(\cos \vartheta^{*}\right)= & \frac{r_{0}^{2}}{4 \beta \gamma^{2}\left(1-\beta^{2} \cos \vartheta^{*}\right)^{2}} X_{\mathrm{C}}[B-C] \\
f_{3}\left(\cos \vartheta^{*}\right)= & \frac{r_{0}^{2}}{4 \beta \gamma^{2}\left(1-\beta^{2} \cos \vartheta^{*}\right)^{2}} X_{\mathrm{S}}[B-C]
\end{aligned}
$$

\section{C.2 Integral cross section}

As mentioned in Sect. 3, (43)-(44), the angular region is restricted to

$$
\begin{aligned}
& 0 \leq \vartheta^{*} \leq \frac{\pi}{2} \\
& 0 \leq \varphi \leq 2 \pi .
\end{aligned}
$$

The integration of the differential annihilation cross section (C.10) over the azimutal angle $\varphi$ yields:

$$
\begin{aligned}
\int_{0}^{2 \pi} \frac{\mathrm{d} \sigma}{\mathrm{d} \Omega} \mathrm{d} \varphi= & 2 \pi f_{1}\left(\cos \vartheta^{*}\right) \\
= & \frac{\pi r_{0}^{2}}{2 \beta \gamma^{2}\left(1-\beta^{2} \cos \vartheta^{*}\right)^{2}}\{[A+B+C+D] \\
& \left.+X_{\mathrm{L}}[-A+B+C-D]+X_{\mathrm{T}}[A-D]\right\} .
\end{aligned}
$$

By integrating over the cosine of the polar angle $\vartheta^{*}$ I obtain three integrals of the following type:

$$
\begin{aligned}
& k_{\nu}\left(\cos \vartheta^{*}\right)=\int_{0}^{\cos \vartheta^{*}} \frac{\cos ^{\nu} \vartheta^{*^{\prime}}}{\left(1-\beta^{2} \cos ^{2} \vartheta^{*^{\prime}}\right)^{2}} \mathrm{~d} \cos \vartheta^{*^{\prime}} \\
& (\nu=0,2,4) .
\end{aligned}
$$

These three integrals are:

$$
\begin{aligned}
& k_{0}\left(\cos \vartheta^{*}\right)=\frac{X}{2}+\frac{Y}{4 \beta} \\
& k_{2}\left(\cos \vartheta^{*}\right)=\frac{X}{2 \beta^{2}}-\frac{Y}{4 \beta^{3}} \\
& k_{4}\left(\cos \vartheta^{*}\right)=\frac{\cos \vartheta^{*}}{\beta^{4}}+\frac{X}{2 \beta^{4}}-\frac{3 Y}{4 \beta^{5}},
\end{aligned}
$$

with the two abbreviations

$$
\begin{gathered}
X \equiv X\left(\cos \vartheta^{*}\right)=\frac{\cos \vartheta^{*}}{1-\beta^{2} \cos ^{2} \vartheta^{*}} \\
Y \equiv Y\left(\cos \vartheta^{*}\right)=\ln \frac{1+\beta \cos \vartheta^{*}}{1-\beta \cos \vartheta^{*}} .
\end{gathered}
$$

Define

$$
\begin{aligned}
& a \equiv a\left(\cos \vartheta^{*}\right)=\int_{0}^{\cos \vartheta^{*}} \frac{A}{\left(1-\beta^{2} \cos \vartheta^{*^{\prime}}\right)^{2}} \mathrm{~d} \cos \vartheta^{*^{\prime}} \\
& b \equiv b\left(\cos \vartheta^{*}\right)=\int_{0}^{\cos \vartheta^{*}} \frac{B}{\left(1-\beta^{2} \cos \vartheta^{*^{\prime}}\right)^{2}} \mathrm{~d} \cos \vartheta^{*^{\prime}} \\
& c \equiv c\left(\cos \vartheta^{*}\right)=\int_{0}^{\cos \vartheta^{*}} \frac{C}{\left(1-\beta^{2} \cos \vartheta^{*^{\prime}}\right)^{2}} \mathrm{~d} \cos \vartheta^{*^{\prime}} \\
& d \equiv d\left(\cos \vartheta^{*}\right)=\int_{0}^{\cos \vartheta^{*}} \frac{D}{\left(1-\beta^{2} \cos \vartheta^{*^{\prime}}\right)^{2}} \mathrm{~d} \cos \vartheta^{*^{\prime}} .
\end{aligned}
$$

I can then express the $a, b, c, d$ as linear combinations of the $k_{\nu}$ :

$$
\begin{aligned}
a & =\frac{\beta^{2}}{\gamma^{2}}\left[2 k_{0}-2 k_{2}+k_{4}\right] \\
b & =\beta^{2}\left[k_{0}-k_{2}\right] \\
c & =\beta^{2}\left[k_{2}-k_{4}\right] \\
d & =\frac{1}{\gamma^{2}} k_{0} .
\end{aligned}
$$

I finally obtain

$$
\begin{aligned}
a+b+c+d= & \left(1+2 \beta^{2}-2 \beta^{4}\right) k_{0} \\
& +\left(-2 \beta^{2}+2 \beta^{4}\right) k_{2}-\beta^{4} k_{4} \\
= & -\cos \vartheta^{*}-\frac{1}{\gamma^{4}} X+\frac{3-\beta^{4}}{2 \beta} Y \\
-a+b+c-d= & \left(-1+2 \beta^{4}\right) k_{0}+\left(2 \beta^{2}-2 \beta^{4}\right) k_{2} \\
& +\left(-2 \beta^{2}+\beta^{4}\right) k_{4} \\
= & \frac{1}{\beta^{2}}\left\{\left(-2+\beta^{2}\right) \cos \vartheta^{*}-\frac{1+\beta^{4}}{\gamma^{2}} X\right. \\
& \left.+\frac{3-3 \beta^{2}+\beta^{4}+\beta^{6}}{2 \beta} Y\right\} \\
a-d= & \frac{1}{\gamma^{2}}\left\{\left(-1+2 \beta^{2}\right) k_{0}\right. \\
& \left.-2 \beta^{2} k_{2}+\beta^{2} k_{4}\right\} \\
= & \frac{1}{2 \beta^{2} \gamma^{2}}\left\{2 \cos \vartheta^{*}+\frac{1-2 \beta^{2}}{\gamma^{2}} X\right. \\
& \left.-\frac{3+2 \beta^{2}}{2 \beta \gamma^{2}} Y\right\} \\
b-c= & \beta^{2}\left\{k_{0}-2 k_{2}+k_{4}\right\} \\
= & \frac{1}{2 \beta^{2}}\left\{2 \cos \vartheta^{*}+\frac{1}{\gamma^{4}} X-\frac{3+\beta^{2}}{2 \beta \gamma^{2}} Y\right\} .
\end{aligned}
$$




\section{C.3 Total cross section}

For the calculation of the total cross section one needs the complete integrals over phase space ((C.24)-(C.27) with $\left.\cos \vartheta^{*}=1\right)$ :

$$
\begin{aligned}
{[a+b+c+d](1)=} & -2+\beta^{2}+\frac{3-\beta^{4}}{2 \beta} Z(\beta) \quad(\mathrm{C} .36) \\
{[-a+b+c-d](1)=} & \frac{1}{\beta^{2}}\left\{-3+\beta^{2}-\beta^{4}\right. \\
& \left.+\frac{3-3 \beta^{2}+\beta^{4}+\beta^{6}}{2 \beta} Z(\beta)\right\} \quad(\mathrm{C} .37) \\
{[a-d](1)=} & \frac{1}{2 \beta^{2} \gamma^{2}}\left\{3-2 \beta^{2}-\frac{3+2 \beta^{2}}{2 \beta \gamma^{2}} Z(\beta)\right\} \\
{[b-c](1)=} & \frac{1}{2 \beta^{2}}\left\{3-\beta^{2}-\frac{3+\beta^{2}}{2 \beta \gamma^{2}} Z(\beta)\right\} \\
\text { with } \quad Z(\beta) \equiv & \ln \frac{1+\beta}{1-\beta} .
\end{aligned}
$$

The total annihilation cross section thus depends both on the longitudinal $\left(X_{\mathrm{L}}=P_{n 3} Q_{n 3},(\mathrm{C} .8)\right)$ as well as on the transverse polarisation components $\left(X_{\mathrm{T}}=P_{n 1} Q_{n 1}\right.$ $\left.+P_{n 2} Q_{n 2},(\mathrm{C} .5)\right)$ of the electron and the positron.

$$
\begin{aligned}
\sigma_{\text {Ann }}= & \frac{\pi r_{0}^{2}}{2 \beta \gamma^{2}}\left\{\left[-2+\beta^{2}+\frac{3-\beta^{4}}{2 \beta} Z(\beta)\right]\right. \\
& +\frac{X_{\mathrm{L}}}{\beta^{2}}\left[-3+\beta^{2}-\beta^{4}+\frac{3-3 \beta^{2}+\beta^{4}+\beta^{6}}{2 \beta} Z(\beta)\right] \\
& \left.+\frac{X_{\mathrm{T}}}{2 \beta^{2} \gamma^{2}}\left[3-2 \beta^{2}-\frac{3+2 \beta^{2}}{2 \beta \gamma^{2}} Z(\beta)\right]\right\} .
\end{aligned}
$$

\section{C.4 Generation of the polar angle $\vartheta^{*}$}

Generate a uniformly distributed random number $G_{1}^{\mu} \equiv G_{1}^{\mu}\left(\cos \vartheta^{*}\right),(\mu=1, \ldots, m)$, in the interval $(0,1]$ and demand:

$$
\begin{aligned}
G_{1}^{\mu}\left(\cos \vartheta^{*}\right) & =\frac{\int_{0}^{\cos \vartheta^{*}} \int_{0}^{2 \pi} \frac{\mathrm{d} \sigma}{\mathrm{d} \Omega}\left(\cos \vartheta^{*^{\prime}}, \varphi\right) \mathrm{d} \varphi \mathrm{d} \cos \vartheta^{*^{\prime}}}{\int_{0}^{1} \int_{0}^{2 \pi} \frac{\mathrm{d} \sigma}{\mathrm{d} \Omega}\left(\cos \vartheta^{*^{\prime}}, \varphi\right) \mathrm{d} \varphi \mathrm{d} \cos \vartheta^{*^{\prime}}} \\
= & \frac{f_{1}\left(\cos \vartheta^{*}\right)}{f_{1}(1)}
\end{aligned}
$$

By iteration (for example by Newton's method) one obtains the correctly distributed random value of $\cos \vartheta^{*}$ :

$$
\cos \vartheta^{*}=\cos \vartheta_{\mu}^{*}
$$

\section{C.5 Generation of the azimuthal angle $\varphi$}

By inserting $\cos \vartheta^{*}=\cos \vartheta_{\mu}^{*}$ into the differential cross section (C.10) one obtains the conditional differential cross section

$$
\begin{aligned}
\frac{\mathrm{d} \sigma}{\mathrm{d} \Omega}\left(\cos \vartheta_{\mu}^{*}, \varphi\right)= & f_{1}\left(\cos \vartheta_{\mu}^{*}\right) \\
& +f_{2}\left(\cos \vartheta_{\mu}^{*}\right) \cos 2 \varphi+f_{3}\left(\cos \vartheta_{\mu}^{*}\right) \sin 2 \varphi
\end{aligned}
$$

Generate a second uniformly distributed random number $G_{2}^{\mu} \equiv G_{2}^{\mu}(\varphi)$ in the interval $(0,1]$ and demand:

$$
\begin{aligned}
G_{2}^{\mu}(\varphi)= & \frac{\int_{0}^{\varphi} \frac{\mathrm{d} \sigma}{\mathrm{d} \Omega}\left(\cos \vartheta_{\mu}^{*}, \varphi^{\prime}\right) \mathrm{d} \varphi^{\prime}}{\int_{0}^{2 \pi} \frac{\mathrm{d} \sigma}{\mathrm{d} \Omega}\left(\cos \vartheta_{\mu}^{*}, \varphi^{\prime}\right) \mathrm{d} \varphi^{\prime}} \\
= & \frac{1}{2 \pi}\{\varphi \\
& +\frac{f_{2}\left(\cos \vartheta_{\mu}\right)}{2 f_{1}\left(\cos \vartheta_{\mu}\right)} \sin 2 \varphi \\
& \left.+\frac{f_{3}\left(\cos \vartheta_{\mu}\right)}{2 f_{1}\left(\cos \vartheta_{\mu}\right)}(1-\cos 2 \varphi)\right\} .
\end{aligned}
$$

By iteration one obtains the azimuthal angle

$$
\varphi=\varphi_{\mu} .
$$

\section{References}

1. C.S. Wu, E. Ambler, R.W. Hayward, D.D. Hoppes, R.P. Hudson, Phys. Rev. 105, 1413 (1957)

2. R.L. Garwin, L.M. Lederman, M. Weinrich, Phys. Rev. 105, 1415 (1957)

3. J. Friedman, V. Telegdi, Phys. Rev. 105, 1415 (1957)

4. L.A. Page, M. Heinberg, Phys. Rev. 106, 1220 (1957)

5. S.L. Glashow, Nucl. Phys. 22, 579 (1961)

6. S. Weinberg, Phys. Rev. Lett. 19, 1264 (1967)

7. A. Salam, in Elementary Particle Theory, Proc. Nobel Symposium held 1968 at Lerum, Sweden (Wiksell, Stockholm, 1968), ed. by N. Svartholm, Almqvist, chap. Weak and Electromagnetic Interactions, pp. 367-377

8. L. Michel, Proc. Phys. Soc. A 63, 514 (1950)

9. K. Mursula, F. Scheck, Nucl. Phys. B 253, 189 (1985)

10. W. Fetscher, H.J. Gerber, K.F. Johnson, Phys. Lett. B 173, $102(1986)$

11. W.-M. Yao et al., J. Phys. G 33, 1 (2006)

12. W. Fetscher, Phys. Rev. D 42, 1544 (1990)

13. W. Fetscher, Complete determination of the weak interaction in muon decay and comparison with the standard model, in Neutrino '86 (Singapore, World Scientific, 1986), ed. by T. Kitagaki, H. Yuta, pp. 600-609

14. W. Fetscher, H.J. Gerber, in Precision Tests of the Standard Electroweak Model, Vol. 14 of Advanced Series on Directions in High Energy Physics (P. Langacker, World Scientific, Singapore, 1995), Chap. Precision measurements in muon and tau decays, pp. 657-705

15. T. Kinoshita, A. Sirlin, Phys. Rev. 108, 844 (1957)

16. F. Scheck, Phys. Rep. 44, 187 (1978) 
17. H. Burkard et al., Phys. Lett. B 160, 343 (1985)

18. N. Danneberg et al., Phys. Rev. Lett. 94, 021802 (2005)

19. A. Buhler et al., Phys. Lett. 7, 368 (1963)

20. F. Corriveau et al., Phys. Rev. D 24, 2004 (1981)

21. H. Burkard et al., Phys. Lett. B 150, 242 (1985)

22. L.A. Page, Rev. Mod. Phys. 31, 759 (1959)

23. W.H. McMaster, Rev. Mod. Phys. 33, 8 (1961)

24. H. Mahler, Messung der Longitudinalpolarisation der Positronen aus dem Zerfall positiver Myonen, $\mathrm{PhD}$ thesis, ETH Zürich, 1980

25. I.C. Barnett et al., Nucl. Instrum. Methods A 455, 329 (2000)

26. F. Corriveau, Mesure de la polarisation transversale des positrons dans la désintégration de muons positifs polarisés, PhD thesis, ETH Zürich, 1985
27. W. Fetscher et al., (to be published)

28. M. Fischer, S. Groote, J.G. Körner, M. C.Mauser, Phys. Rev. D 67, 113008 (2003)

29. TWIST, J. Musser et al., Phys. Rev. Lett. 94, 101805 (2005) [hep-ex/0409063]

30. TWIST, A. Gaponenko et al., Phys. Rev. D 71, 071101 (2005) [hep-ex/0410045]

31. I. Beltrami et al., Phys. Lett. B 194, 326 (1987)

32. B. Jamieson et al., Phys. Rev. D 74, 072007 (2006)

33. A. Jodidio et al., Phys. Rev. D 37, 237 (1988)

34. X. Morelle et al., Measuring the Michel parameter $\xi^{\prime \prime}$ in polarized muon decay, Vol. 570, 2001, Prepared for 14th International Spin Physics Symposium (SPIN 2000), Osaka, Japan, 16-21 Oct 2000 